\title{
Arqueología del cielo. Orientaciones astronómicas en edificios protohistóricos del sur de la Península Ibérica
}

\author{
Archaeology of the sky. Astronomical orientations in Protohistoric buildings of the south \\ of the Iberian Peninsula
}

\author{
César Esteban (*) \\ José Luis Escacena Carrasco (**)
}

\section{RESUMEN}

Presentamos un análisis arqueoastronómico de la orientación de seis santuarios y edificios de carácter público protohistóricos en sitios arqueológicos situados en el mediodía de la Península Ibérica (Coria del Río, El Carambolo, Saltillo, Mesa de Setefilla, Tejada la Vieja y El Oral). El estudio, el primero de este tipo que se realiza sobre una muestra de santuarios del Hierro Antiguo en esta área geográfica, se basa en la medida precisa de las orientaciones definidas por los edificios y el análisis del horizonte que les rodea y revela claros vínculos astronómicos. En particular, los edificios de culto muestran unas características similares y una orientación hacia un acimut de $55^{\circ}$. Las regularidades encontradas parecen sugerir la posible existencia de credos y rituales religiosos relacionados con posiciones singulares de los astros principales visibles a simple vista, como el Sol, la Luna o Venus.

\footnotetext{
ABSTRACT

We present an archaeoastronomical analysis of the orientations of six protohistoric sanctuaries and public buildings located in archaeological sites of the south of the Iberian Peninsula (Coria del Río, El Carambolo, Saltillo, Mesa de Setefilla, Tejada la Vieja and El Oral). This study, the first of its kind conducted on a sample of Early Iron Age sanctuaries in this geographic area, is

(*) Instituto de Astrofísica de Canarias. C/ Vía Láctea s/n. 38205 La Laguna. Tenerife. España. Correo e: cel @)iac.es

(**) Dpto. de Prehistoria y Arqueología, Facultad de Geografía e Historia, Universidad de Sevilla. C/ María de Padilla s/n. 41004 Sevilla. España. Correo e.: escacena@us.es

Recibido 5-I-2012; aceptado 12-IV-2012.
}

based on the accurate measurement of the orientations defined by the buildings and the analysis of the horizon around them and reveals clear astronomical relations. In particular, religious buildings show similar characteristics and orientation towards an azimuth of $55^{\circ}$. The regularities found seem to suggest the existence of religious beliefs and rituals linked to singular positions of the brightest celestial bodies as the Sun, Moon or Venus.

Palabras clave: Arqueoastronomía; Tartessos; Fenicios/ Colonización fenicia; Religión; Templos; Urbanismo; Orientación astronómica; Baal; Astarté.

Key words: Archaeoastronomy; Tartessos; Phoenicians/ Phoenician colonisation; Religion; Temples; Town planning; Astronomical orientation; Baal; Astarte.

\section{INTRODUCCIÓN}

El papel de la arqueoastronomía como ramificación de los estudios arqueológicos tiene cada vez una mayor aceptación a nivel académico. Durante los últimos veinte años ha aparecido un número creciente de trabajos de esta temática en las revistas especializadas españolas de Arqueología y Prehistoria (López Plaza et al. 1991-92; Iwaniszewski 1994; Esteban y Cortell 1997; Cerdeño et al. 2001-02; Esteban 2002; Cerdeño et al. 2004; Cerdeño et al. 2006; Esteban y Moret 2006; Rodríguez et al. 2006). M. Cerdeño y G. Rodríguez (2009) coordinaron un volumen especial sobre arqueoastronomía que incluye catorce artículos en una de las revistas más relevantes del área. 
En la actualidad, los estudios de arqueoastronomía cuentan en España con una trayectoria aún breve, aunque con un grado de desarrollo y aceptación completamente equiparable a los de nuestro entorno europeo (Esteban 2003; Cerdeño et al. 2006; Belmonte 2009). Las dos líneas de investigación mayoritarias han sido el análisis de la orientación de los megalitos del sur y suroeste de la Península Ibérica y las Islas Baleares (López Plaza et al. 1991-92; Hoskin 2001, 2006; González García 2009) y las relaciones astronómicas en santuarios y necrópolis de culturas de la Edad del Hierro como la celtibérica (Almagro-Gorbea y Gran-Aymerich 1991; Armentia et al. 1993; Baquedano y Escoraza 1998; Cerdeño et al. 2001-02; Cerdeño et al. 2004; Cerdeño et al. 2006; Cerdeño y Rodríguez 2009) e ibérica (Esteban y Cortell 1997; Esteban 2002; Esteban y Moret 2006).

Los aspectos astronómicos del mundo religioso y funerario del Hierro Antiguo hispano han sido escasamente investigados. M. L. Ramos Sainz (1986: 32) ya indicó que la mayoría de las tumbas del período fenicio arcaico en la Península Ibérica se disponen con sus ejes longitudinales cercanos a la línea este-oeste. Esta misma autora señaló que las necrópolis de Trayamar y de Puente de Noy orientan siempre sus tumbas de cámara con la puerta hacia el este. Accesos dispuestos hacia el oriente también se encuentran en los hipogeos de Villaricos (Belmonte 1999: 186) y de Málaga (González García et al. 2007), según una distribución que muestra dos picos bien diferenciados relacionables respectivamente con el orto solar en el solsticio de invierno y con los equinoccios. Uno de nosotros (Esteban 2012) ha analizado el patrón de orientaciones de las sepulturas en fosa de las necrópolis de La Angorrilla (Alcalá del Río, Sevilla) y de Cerrillo Blanco (Porcuna, Jaén), ambas fechadas entre los siglos VII y VI a. C., encontrando en los ejes mayores de las tumbas un patrón de orientaciones análogo, que cubre el rango de ángulos donde se producen los ortos $\mathrm{u}$ ocasos solares o lunares. El otro autor (Escacena 2007, 2009) ha tratado las relaciones astronómicas de la orientación de los santuarios de Coria del Río y del Carambolo, en el Bajo Guadalquivir, más que nada para ocuparse de los posibles ritos solares asociados a los templos y a sus altares entre los siglos IX y VI a.C.

En este trabajo presentamos un estudio arqueoastronómico de 6 santuarios y posibles lu-

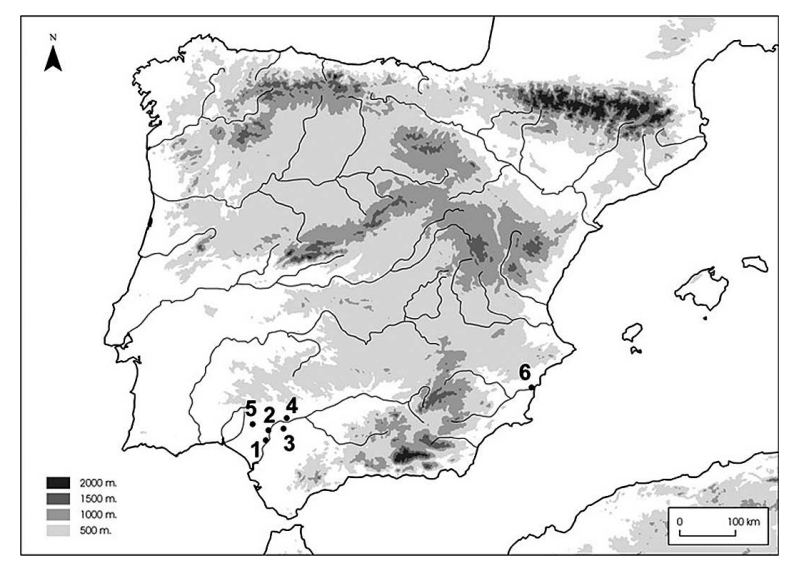

Fig. 1. Mapa de situación de los edificios protohistóricos del sur de la Península Ibérica estudiados. Provincia de Sevilla: 1 Caura (Coria del Río), 2 Carambolo (Sevilla), 3 Saltillo (Carmona), 4 Mesa de Setefilla (Lora del Río); provincia de Huelva: 5 Tejada la Vieja (Escacena del Campo); provincia de Alicante: 6 el Oral (San Fulgencio).

gares de culto, de los cuales 4 corresponden al Hierro Antiguo (siglos IX a VI a.C.) y 2 al Hierro Reciente (siglos V a IV a.C.). Se sitúan en el valle del Guadalquivir, excepto uno, localizado en la desembocadura del río Segura, en la provincia de Alicante (Fig. 1). Nuestro objetivo principal ha sido explorar las posibles relaciones astronómicas de la orientación de los edificios y del horizonte visible desde ellos. Se consideran los dos yacimientos mas recientes como interesantes elementos de comparación, bien por su cercanía espacial (caso del 'edificio público' de Tejada la Vieja), bien por compartir elementos del primer grupo (el altar tipo de piel de toro del santuario ibérico de El Oral). Somos conscientes de que los yacimientos estudiados son solo una muestra limitada de los posibles edificios de culto del Hierro Antiguo conocidos en el sur peninsular. Nuestra intención es ampliar la muestra en el futuro para que las conclusiones alcancen el mayor peso estadístico posible.

\section{LA TOMA DE DATOS: DESCRIPCIÓN DE LA FASE DE CAMPO}

La fase de campo se llevó a cabo entre el 22 y el 25 de abril de 2006 (excepto en el Oral, véase apar-

Trab. Prehist., 70 N. ${ }^{\circ}$ 1, enero-junio 2013, pp 114-139, ISSN: 0082-5638 doi: $10.3989 /$ tp.2013.12105 
tado 4) y contó en la mayoría de los casos con la colaboración y el asesoramiento de los arqueólogos responsables de la excavación de los yacimientos o que formaron parte del equipo en su día. Se utilizó una brújula de precisión, un clinómetro de mano, un teodolito portátil, un aparato de posicionamiento global $(G P S)$ y una cámara fotográfica digital. La metodología de la toma de datos se detalla en Esteban y Delgado (2005) y en Esteban y Moret (2006) por lo que solo la resumiremos.

El GPS sirvió para obtener las coordenadas geográficas de los yacimientos, así como para cronometrar las medidas de la posición del Sol necesarias para corregir el nivel cero de los ángulos horizontales proporcionados por el teodolito. Este instrumento óptico determinó las coordenadas horizontales (acimut y altura) de los elementos topográficos del horizonte que rodea a los sitios analizados. La precisión de este aparato es de $0,045^{\circ}$ sexagesimales (unos 2,7 minutos de arco, $1 / 10$ del diámetro solar aproximadamente) para ambos ejes. Las medidas de altura cercanas al horizonte se corrigieron del efecto de la refracción atmosférica, la cual hace que la altura aparente de un astro tienda a ser mayor que la real (Esteban y Moret 2006: 170-171).

El teodolito mide ángulos horizontales respecto a un origen de coordenadas arbitrario cuya posición varía cada vez que cambiamos de lugar el instrumento. Sin embargo, para el estudio necesitamos manejar ángulos horizontales referidos al norte geográfico, que denominamos acimutes. Por ello debemos conocer la diferencia angular entre el norte geográfico y el origen de los ángulos horizontales del teodolito en la posición en que lo estemos utilizando. Determinamos este ángulo de corrección centrando el disco solar en el retículo del visor en varias ocasiones (casi siempre tres) durante la visita al yacimiento. Para cada observación, y cronometrando la acción con el $G P S$, calculamos el acimut real del Sol a partir de los datos de coordenadas ecuatoriales diarias, publicados en el Almanaque Náutico del Observatorio de San Fernando (Cádiz). El promedio de las diferencias entre el ángulo horizontal medido con el teodolito y el acimut calculado para cada una de las medidas individuales del centro del disco solar nos proporcionará el ángulo de corrección.

El teodolito proporciona demasiada exactitud para calcular la orientación de las construcciones de los yacimientos por lo que estas se midieron con una brújula de precisión cuya incertidumbre es del orden de $1^{\circ}$. Los ángulos horizontales que se obtienen con la brújula están referidos al norte magnético, por lo que también fue necesario obtener la declinación magnética, que corresponde al ángulo de corrección que hay que aplicar a las lecturas de la brújula para lograr el acimut. Dicho valor se consiguió comparando el ángulo horizontal medido con la brújula para varias cotas topográficas (generalmente campanarios de poblaciones cercanas y cumbres de montañas) con los acimutes proporcionados por el teodolito (calibrado tal y como describimos) para esas mismas cotas o por líneas definidas entre la posición del yacimiento y dichas cotas trazadas sobre mapas del Servicio Geográfico del Ejército o del Instituto Geográfico Nacional a escala 1:50.000 ó 1:100.000.

A partir de las coordenadas horizontales -acimut $(A)$ y altura $(h)$ - obtenidas para un punto cualquiera del horizonte, y conocida la latitud del lugar, podemos calcular la declinación celeste (1) ( $\delta)$ del astro que tiene su orto $u$ ocaso por dicho punto a través de una sencilla ecuación de transformación de coordenadas (Esteban y Moret 2006: 171, ecuación 5).

\section{SANTUARIOS Y POSIBLES LUGARES DE CULTO EN EL GUADALQUIVIR INFERIOR}

\subsection{El templo de Caura (Coria del Río, Sevilla)}

Las intervenciones arqueológicas en el Cerro de San Juan de Coria del Río, cabezo en el que se ubicó la antigua Caura, han revelado que, tras una acumulación de niveles prehistóricos, el asentamiento del primer milenio a.C. incluyó una pequeña comunidad fenicia (Escacena 2001). A esta fase corresponden las primeras construcciones con cimientos-zócalos de piedra y alzado de adobes, además de la expansión del hábitat mediante la fundación de un templo y de un barrio dispuesto en damero a su alrededor (Escacena e Izquierdo 2001).

(1) La declinación corresponde al ángulo sexagesimal entre un punto de la esfera celeste y el ecuador celeste medido a lo largo del círculo mayor que pasa por los polos celestes y dicho punto (Aparicio et al. 2000: 27, fig.1.2). 
La superposición de cinco estructuras con similar diseño sugiere la identidad en su función, de manera que la identificación de una de ellas como santuario permite extender ese mismo papel a las demás. Desde la más antigua (I) a la más reciente (V) se produjeron algunas modificaciones, pero nunca tan profundas como para pensar en su abandono como lugar de culto. Sólo el edificio más reciente, cuya superficie se redujo, podría arrojar algunas dudas al respecto. Aún habiéndose levantado como recinto sagrado, al final de sus días pudo desempeñar un papel no religioso. La construcción que muestra más claramente su función cultual corresponde al Santuario III del siglo VII a.C. En ella se documentó una estancia con suelo rojo adosada al muro perimetral del templo, con un banco de barro paralelo a esta misma pared exterior y un altar en el centro en forma de piel de toro (Escacena e Izquierdo 2000).

Los altares taurodérmicos son ya bien conocidos en la Protohistoria hispana y caracterizan a centros de culto tanto de carácter rural como urbano. Valga como ejemplo, por inaugurar la serie de hallazgos, el santuario de Cancho Roano, en Extremadura (Celestino 1994, 1997, 2008). El templo de Caura pertenece al modelo urbano y la topografía de su emplazamiento, cercano a la subida más fácil al cabezo, insinúa su proximidad a una posible puerta de la ciudad.

El santuario de Coria del Río fue un recinto abierto delimitado por un muro. Se desconoce aún si pudo tener su acceso principal por la fachada oriental. Es posible que dispusiera de otro en la cara opuesta, donde se constata un retranqueo de la pared exterior para alojar un posible pórtico. Pero esta alternativa no es determinable porque estos vanos de entrada y salida no se reflejaron en la cimentación de piedra, que es la única parte conservada del perímetro del edificio. Lo mismo ocurre en las casas vecinas. Con ello se querían evitar zonas especialmente débiles en los fundamentos de la obra (Escacena e Izquierdo 2001: 146). El templo pudo disponer por tanto de dos puertas, una al oriente y otra a poniente.

La estancia más sagrada corresponde a la capilla del Santuario III donde apareció el altar en taurodermis. Es un pequeño recinto que debió estar cubierto, aunque sin paredes en sus cuatro costados. La presencia de fuego sobre el ara haría imprescindible una buena ventilación, con lo que quizás solo un cobertizo lo protegiera de las in- clemencias meteorológicas. La ausencia de muros en sus lados sur y oeste refuerza esta hipótesis. Sobre su suelo de tierra apisonada y pintada de rojo se levantó un banco de unos $10 \mathrm{~cm}$ adosado a la tapia que contornea el santuario, en este caso, también de barro e igualmente pintado de rojo como el pavimento.

Desde su construcción, el altar no quedó paralelo a la pared del templo ni al eje longitudinal de la capilla. Este rasgo sobre su orientación no está certificado. De hecho, no se pudo localizar el muro externo del santuario en sus proximidades porque parece correr en la misma vertical que el cimiento del Santuario IV, sí localizado pero no levantado durante el proceso de excavación. Sin embargo, la orientación del eje longitudinal del altar de barro del Santuario III sería la misma que la del primer edificio (Santuario I), aunque esta fase del templo se conoce solo por una esquina de la construcción. La evolución topográfica y urbanística del complejo religioso, probablemente sujeta a la necesidad de ampliación constante de la anchura de la calle aledaña, podría explicar la falta de una superposición exacta de los edificios. De ahí que los cuatro templos superiores exhiban una orientación ligeramente distinta de la que puede desprenderse del escaso tramo de cimentación conocido aún del templo que inauguró la serie en el siglo VIII a.C.

En su momento de uso más antiguo (fase A), el altar dispuso en su lado oriental de una protuberancia que imitaba la prolongación de la piel correspondiente al cuello del bóvido, y en este apéndice de una oquedad para colocar una muestra de la sangre de la víctima inmolada. Por eso, este flanco oriental puede considerarse el sector más importante del altar desde el punto de vista ritual y simbólico. Que se colocara hacia el este puede ser, pues, una clave a tener en cuenta en nuestro análisis. De hecho, también en esta zona, aunque fuera del ara propiamente dicha, se documentó un círculo de tierra oscura interpretado como hueco para embutir la asherah o árbol sagrado (Escacena e Izquierdo 2001: 134).

La función religiosa de este edificio de Caura se sustenta en otros hallazgos propios de ambientes templarios: dos escarabeos de los siglos VII-VI a.C. y algunos fragmentos de cáscaras de huevos de avestruz (Conde et al. 2005). Con estos datos, y con base en una sugerente hipótesis propuesta por M. Belén (1993) antes de descubrirse este santua-

Trab. Prehist., 70 N. ${ }^{\circ}$ 1, enero-junio 2013, pp 114-139, ISSN: 0082-5638 doi: $10.3989 /$ tp.2013.12105 
rio, hoy puede sostenerse su posible consagración a una divinidad protectora de los marineros (Baal Saphon). Su ubicación en este enclave estaría basada en que el asentamiento controlaba entonces la desembocadura del Guadalquivir en el golfo tartésico (Arteaga et al. 1995: 109).

A fin de proteger las construcciones exhumadas, los restos del santuario de Coria del Río se volvieron a enterrar después de su hallazgo en 1997-98, mientras que el altar se extrajo para su restauración en el Instituto Andaluz de Patrimonio Histórico. Como las estructuras no se han podido medir directamente optamos por medir elementos arquitectónicos modernos que estuvieran al descubierto y que figurasen en la planimetría detallada disponible sobre el yacimiento. Es el método usado generalmente para determinar después la orientación de los elementos arqueológicos de interés astronómico. Los planos precisan la disposición relativa de los elementos arquitectónicos, pero no la posición absoluta de los conjuntos respecto al norte geográfico, ya que en general marcan el norte magnético. Esto dificulta, y a veces imposibilita, utilizar la planimetría publicada para trabajos arqueoastronómicos precisos (Esteban 2009). En el caso del santuario de Coria del Río, se midió con la brújula la orientación del muro perimetral del Instituto de Educación Secundaria (IES Caura), ubicado sobre el yacimiento, al noreste de la zona excavada. El error estimado para la orientación de dicho muro es de $1^{\circ}$. Posteriormente, se determinó el ángulo formado entre el eje del altar y el muro del IES Caura sobre la planimetría y se sumó al acimut obtenido con la brújula. Se calculó además la declinación magnética apropiada comparando los ángulos de elementos topográficos del horizonte local obtenidos con la brújula y los acimutes definidos en planos detallados de la zona. De esta manera, y considerando según hemos adelantado que la dirección relevante del altar sería la oriental, el acimut del eje mayor de este elemento sería de $55,5^{\circ}$, al que le podemos asignar una incertidumbre de unos $2^{\circ}$ (Fig. 2).

La presencia de construcciones modernas sobre el cabezo impidió llevar a cabo una autopsia directa del horizonte oriental. De todas formas, desde el talud del yacimiento que cae a plomo sobre el Guadalquivir se pudo comprobar que el horizonte lejano es muy plano y de una altura (h) muy baja. Suponiendo $h=0^{\circ}$, el valor del acimut encontrado para el altar correspondería

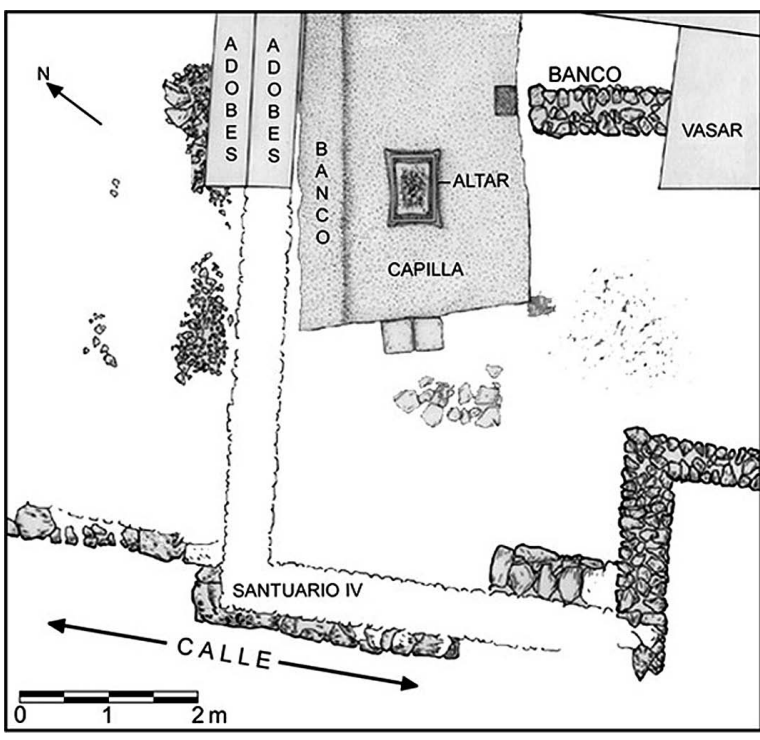

Fig. 2. Plano de las fases III y IV del santuario tartésico de Coria del Río (Sevilla) con sus distintos elementos.

con el punto del horizonte donde se produce el orto de un astro con una declinación del orden de $+26,4^{\circ} \pm 1,5^{\circ}$, ligeramente al norte del orto solar del solsticio de verano. Según las evidencias arqueológicas, hubo construcciones al norte y al este del santuario coetáneas a este último, que obstruyeron la observación directa de la vista oriental desde el templo en los momentos de construcción del Santuario III. Por el contrario, al estar situado el edificio en la pendiente occidental del promontorio, en la dirección del puerto de la antigua ciudad de Caura, el horizonte occidental tuvo una mayor probabilidad de estar despejado, como ocurre aún en la actualidad.

Como se ve en la figura 2, el banco de barro situado inmediatamente al norte del altar presenta la misma orientación que este, mientras que el tramo de muro (posiblemente un banco) que se localiza al sur es exactamente perpendicular. Las otras paredes sincrónicas del altar no comparten su orientación ni tampoco son exactamente perpendiculares entre ellas. Sin embargo, es importante indicar que la esquina del edificio de época fundacional (Santuario I) sí parece coincidir con la orientación del altar. Ello indicaría que el acimut de $55,5^{\circ}$ fue el que definió originalmente la disposición del recinto inaugural. En cualquier caso, esta última conclusión se basa en un tramo 
muy pequeño, una esquina del cimiento, conocido hasta ahora del Santuario I.

\subsection{El santuario del Carambolo (Camas, Sevilla)}

La tradición historiográfica que cuenta con más partidarios ha visto en el Carambolo un poblado tartésico de fondos de cabaña circulares u ovales, es decir, un asentamiento indígena fundado antes de la colonización fenicia. Las fechas sostenidas, no hace mucho para sus materiales más característicos, habrían venido a reforzar esta hipótesis (Castro et al. 1996: 198). No obstante, tras las excavaciones iniciadas a raíz del hallazgo del tesoro que ha proporcionado fama al yacimiento, se intuyó la presencia de elementos de carácter sagrado que reflejarían una profunda singularidad del sitio (Carriazo 1973: 292-293). Es decir, la hipótesis de que el Carambolo fuera un centro religioso, más que un asentamiento común, comenzó muy pronto, pero perduró mucho solo en estado potencial. A pesar de las observaciones de Carriazo, fue A. Blanco Freijeiro (1979: 95-96), quien propuso de modo explícito que allí existió un templo, si bien lo consideró tartésico como el asentamiento. Habiendo reconocido por doquier influencias orientales, no reparó en que el exvoto de Astarté del Museo Arqueológico Hispalense, cuyo posible hallazgo en el Carambolo él mismo resolvió (Blanco 1968: n. 5), sugería vínculos fenicios. Pesaba tanto el axioma 'fenicios en la costa/tartesios en el interior', que todo lo de sabor oriental localizado en las tierras andaluzas no litorales se interpretaba como reflejo de la orientalización del mundo indígena, nunca como la presencia directa de colonos semitas. Quedaba lejos en el tiempo, además, la defensa de asentamientos orientales en la comarca de los Alcores por G. Bonsor (1899).

A pesar de la tendencia investigadora imperante, algunos autores vieron en el Carambolo un santuario con sus servicios anejos más que un asentamiento con su templo correspondiente. Allanaron el camino para comprender los descubrimientos recientes, porque evidenciaron el carácter singular y religioso de algunos ajuares cerámicos o la existencia de estructuras de posible uso religioso entre lo excavado por Carriazo
(Belén y Escacena 2002: 169). Otras pesquisas ahondaron en el carácter cúltico de algunas piezas (Izquierdo y Escacena 1998) y en un cambio radical de la función del tesoro, que de joyas reales pasaron a tenerse por atalaje para engalanar toros destinados al sacrificio y ajuar litúrgico del sacerdote encargado del correspondiente rito (Amores y Escacena 2003).

Desde el año 2002, las excavaciones en la parte superior del cabezo han ido confirmando la hipótesis que ve en el lugar un recinto de culto (Fernández Flores y Rodríguez Azogue 2005a, 2005b, 2007; Rodríguez Azogue y Fernández Flores 2005). Según ellas, el edificio comenzó como una humilde estructura rectangular subdividida en tres espacios: un patio de entrada y dos estancias cubiertas al fondo. Desde el exterior una pequeña rampa permitía subir hasta el umbral de la puerta del conjunto, ubicada en la fachada oriental. Aunque ambas capillas del fondo occidental estaban muy destruidas en el momento de su excavación, la meridional conservaba en su centro un altar circular. Hecho con barro amarillento, presentaba hacia el este una estructura muy deteriorada elaborada con el mismo tipo de arcilla. Pudo tener la misma forma que el localizado en la fase $\mathrm{C}$ del santuario extremeño de Cancho Roano (Celestino 2001: 28-30). La datación radiocarbónica lleva esta fase inicial del complejo sacro al siglo IX a.C., algo que la presencia de cerámica fenicia confirma arqueológicamente.

Durante el siglo siguiente el solar ocupado por este pequeño templo se convierte en patio central trasero de un enorme complejo ceremonial de planta de tendencia cuadrada o rectangular. A esta etapa corresponde la construcción de un gran espacio abierto de entrada y de un conjunto de estancias rectangulares al fondo articuladas en torno al patinillo central trasero que antes fuera primer santuario.

Al norte del pequeño patio del fondo y separado de el por un cuarto de servicio alargado, se levantó una capilla con bancos adosados a sus paredes longitudinales, que se pintaron de blanco y rojo. Este último color se aplicó sucesivamente al suelo de la estancia mediante finas capas de pintura. Hacia el fondo de la habitación, a la que se accedía desde el gran acerado de caparazones de moluscos y que se interpreta como capilla de Astarté, se encontró una estructura de adobes, en su mayor parte arrasada por construcciones mo-

Trab. Prehist., 70 N. ${ }^{\circ}$ 1, enero-junio 2013, pp 114-139, ISSN: 0082-5638 doi: $10.3989 /$ tp.2013.12105 
dernas, que se ha interpretado como base de un altar por simetría con la estancia similar situada al sur del patio interior.

Pero la cella mejor conservada es la meridional. Se trata de nuevo de un espacio rectangular separado también del patio interior por un cuarto alargado. De esta forma, el núcleo central del edificio adquiría una simetría casi perfecta. Este espacio, interpretado como capilla para la divinidad masculina (Baal/Melqart), se rodeó asimismo de bancos de adobe. En el centro de esta gran sala se dispuso un altar en forma de piel de toro que apenas levantaba unos centímetros del suelo. Dicha altura solo la consiguió al final de su vida y a base de repintados y retoques, pues en origen era una simple impronta en el pavimento de barro apisonado de la estancia. Recubierto por completo de tinte rojo, conservaba en su centro la espectacular huella del hogar, que en este caso trascendía los límites del ara. En parte semejante al de Caura, el altar taurodérmico del Carambolo es, en cambio, de silueta más esquemática, mucho más plano y de mayor tamaño, en casi todo similar al diseño de los frontiles del tesoro que casi cincuenta años antes apareciera unos $35 \mathrm{~m}$ más al norte.

En atención al exvoto encontrado en este cabezo, hay fuertes razones para asumir la consagración del santuario del Carambolo a la diosa Astarté, lo que no niega en absoluto la celebración en él de cultos dedicados a su compañero divino (Baal/Melqart). De ahí se deduciría su carácter semita, una vinculación étnica y cultural acrecentada por hallazgos como fragmentos de huevos de avestruz, algunos escarabeos y un barco votivo de cerámica con la forma de híppos fenicio (Escacena et al. 2007).

Emplazado al oeste de *Spal $>$ Hispalis (Sevi1la), en uno de los cerros más altos de la cornisa oriental de la meseta del Aljarafe, el santuario ocupaba una elevación singular de la orilla derecha del paleoestuario del Guadalquivir, muy cerca -apenas $10 \mathrm{~km}$ - de su antigua desembocadura en Caura. En consecuencia, si este paisaje es el que la Ora Maritima describe en las bocas del gran río de Tartessos, y si es correcta la identificación de Caura con el Mons Cassius (Belén 1993), este sitio puede corresponder al que Avieno en los mismos versos de su poema denomina Fani Prominens, es decir, el 'promontorio del templo' (Or. Mar. 259-261).
Los excavadores han numerado los distintos santuarios superpuestos del Carambolo al revés que en Caura: ahora la construcción más antigua es el edificio 'Carambolo V' y la más reciente el complejo 'Carambolo I'. Estos santuarios han podido medirse de forma directa para nuestro trabajo. La tarea de campo se centró en tomar la orientación de las estancias más relevantes del complejo: la habitación A-40 o capilla de Baal de las fases Carambolo IV y III, que contenía el altar taurodérmico (UE-2531), y los espacios A-29 y A-46 del momento inicial o Carambolo V. Estos dos últimos, junto con la pequeña sala A-45, formaban el recinto sagrado prístino, siendo A-29 el patio de entrada, A-45 la capilla meridional del fondo y A-46 la capilla septentrional. La figura 3 muestra fotos aéreas de las estructuras indicando los elementos cuya orientación se evaluó. Se usó la brújula de precisión en todos los casos. Del patio de acceso (espacio A-29) se tomó en consideración básicamente la pared meridional, que conserva un banco corrido a todo lo largo de su cara interna. Paralelo a este muro y a su poyo adosado se encuentra el banco del lado septentrional del patio, que sigue la misma orientación. La estancia A-46, al fondo del patio A-29, se interpreta como capilla para la divinidad masculina Baal/Melqart de esta fase del santuario, mientras que la adosada por el norte, mal conservada pero supuestamente paralela a ella, estaría dedicada a Astarté. Al medir las paredes septentrional y meridional de la cella A-46, constatamos que son paralelas. En este edificio inicial consideraremos como orientación representativa del santuario más antiguo (Carambolo V) la definida por estas dos capillas (estancias A-46 y A-45). Esta orientación parece más ajustada al eje de simetría del templo en su conjunto. La declinación magnética se determinó a partir del valor medio de las diferencias entre los ángulos horizontales medidos con la brújula para varias cotas lejanas y sus acimutes, obtenidos estos últimos sobre planos topográficos detallados de la zona.

El análisis arqueoastronómico del Carambolo se basó en la determinación del acimut de los distintos elementos del edificio y en la declinación del astro que tiene su orto hacia el punto del horizonte al que miran las puertas del recinto. El valor de la altura promedio del horizonte se tomó como $h=0^{\circ}$. Como acabamos de señalar, elegimos la dirección oriental debido a que las entradas de

Trab. Prehist., 70, N. ${ }^{\circ}$ 1, enero-junio 2013, pp 114-139, ISSN: 0082-5638 doi: $10.3989 /$ tp.2013.12105 
todas las estancias están abiertas a este flanco. A ello debe sumarse que el hueco para la asherah localizado en la capilla sur del Carambolo III, al pie del altar taurodérmico, también privilegia el este, como en Caura. En el edificio inaugural (Carambolo $\mathrm{V}$ ), las capillas situadas al fondo apuntan a un acimut de $59^{\circ}$ (declinación $\left.+24^{\circ}\right)$, mientras que el ámbito A-29, patio que precede a esas capillas, presenta una orientación ligeramente más al norte: $55,8^{\circ}$. Todos los ángulos anteriores tienen una incertidumbre asociada en torno a $1^{\circ}$. Las paredes y bancos corridos de la estancia A-40, la capilla sur del Carambolo IV y III, proporcionan una orientación promedio de unos $54,6^{\circ}$ (declinación $+27^{\circ}$ ), con una desviación estándar de $2^{\circ}$ (del orden de $1,5^{\circ}$ en declinación). Desgraciadamente, la orientación del eje mayor del gran altar taurodérmico de esta sala, siglado en el proceso de excavación como unidad estratigráfica UE-2531, no pudo medirse por estar tapado cuando se llevó a cabo nuestro trabajo de campo. Sí sabemos que su eje mayor es paralelo a los muros longitudinales de la sala que lo cobija, la A-40 (Fig. 3). Otro ámbito importante que tampoco pudimos medir entonces fue el que se considera capilla de Astarté en las fases Carambolo IV y III (estancia A-1). Este espacio disponía bajo el pavimento rojo de una plataforma de ladrillos crudos que los excavadores han interpretado como base de un posible altar. De cualquier manera, en la fotografía aérea publicada (Fernández Flores y Rodríguez Azogue 2007: 116, fig. 23) se puede comprobar que la pared meridional (la mejor conservada) de la estancia A-1 es paralela a la pared septentrional de A-29, por lo tanto, el acimut de A-1 debe ser cercano a $63,5^{\circ}\left(\right.$ declinación $\left.+20,4^{\circ}\right)$.

Es necesario considerar que el santuario Carambolo $\mathrm{V}$ no estaba en época protohistórica exactamente en la parte más alta del cerro, sino a unos $40 \mathrm{~m}$ de la cumbre en dirección oeste. Según el informe geoarqueológico, la diferencia de altura entre la cima del cabezo y la base del templo sería de unos 3 m (Borja 2010: 197-200). Por lo tanto, una persona situada de pie sobre el suelo de este edificio primitivo, a la entrada del complejo, no vería los ortos de los astros sobre el horizonte lejano, sino sobre la corona del promontorio, situada a una altura aproximada de $h \approx 2^{\circ}$. Considerando este nuevo horizonte, las declinaciones de los astros hacia cuyos ortos apuntan las distintas estancias serían $\mathrm{A}-46=+25,5^{\circ}$ y A- $40=+28,5^{\circ}$.

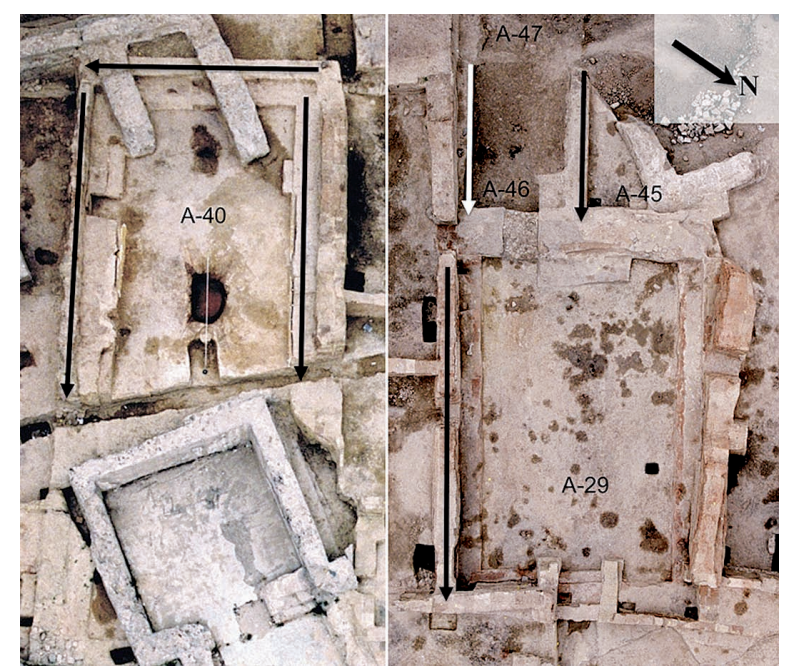

Fig. 3. Vista aérea de las estancias A-40, A-29 y A-46 del santuario tartésico del Carambolo (Sevilla) (Fernández Flores y Rodríguez Azogue 2007: 116, fig. 23) estudiadas en el presente trabajo. Las flechas de trazo más fino indican los elementos cuya orientación se midió con la brújula de precisión. La anchura de las estancias A-40 y A-29 es de unos 5 y $7 \mathrm{~m}$ respectivamente.

Es decir, la variación en declinación en todos los casos sería de unos $1,5^{\circ}$ al norte. La incertidumbre asociada a dichos valores corregidos de la declinación se ha incrementado en $0,7^{\circ}$ al propagar una incertidumbre adicional de $\pm 1^{\circ}$ en la altura.

Finalmente, también medimos algunos elementos topográficos relevantes del horizonte oriental, sobre todo para comprobar la existencia de marcadores astronómicos y/o topográficos como los encontrados en otros santuarios protohistóricos (Esteban 2002; Esteban y Moret 2006). Pero no se encontraron tales relaciones con un interés relevante.

\subsection{El complejo de Saltillo (Carmona, Sevilla)}

Durante el seguimiento de unas obras, y sobre todo durante la posterior excavación de urgencia en un solar en la plaza Marqués de Saltillo, en el barrio de San Blas de Carmona, se hallaron en 1992 unas estructuras arquitectónicas pertenecientes a un complejo edilicio interpretado como lugar de culto. Se localizaron en esos trabajos tres edifi- 
cios superpuestos, fechados por los materiales arqueológicos que contenían entre la segunda mitad del siglo VII a.C., como mucho, y mediados del $\mathrm{V}$ a.C. Según sus características arquitectónicas, todas las construcciones de este recinto muestran rasgos orientales, que en el mundo tartésico hay que atribuir básicamente a la colonización fenicia.

En la fase más arcaica del conjunto pudo documentarse una estancia rectangular de 4,4 x 1,8 $\mathrm{m}$, paredes de adobe y cimientos-zócalos de mampostería pétrea, dispuesta mediante un eje orientado longitudinalmente en sentido este-oeste. Los excavadores denominaron esta habitación 'Ámbito 6'. Parece que se entraba por una puerta que, por los datos recabados durante la excavación, solo pudo estar situada en la esquina que más tarde ocupó un pozo de época romana. La sala contaba con 3 rincones o ángulos internos en los que, encastrados en el suelo rojo, se ubicaban sendas vasijas del tipo conocido como pithos de motivos orientalizantes. Sobre el pavimento se rescataron también otros contenedores cerámicos, entre los que destaca una gran orza fabricada a mano y las posibles tapaderas de los 3 recipientes decorados, así como un juego de cucharas de marfil que forma en conjunto las patas delanteras y traseras de un animal de doble pezuña (Belén et al. 1997; Belén 2001).

Tan singular estructura $y$, sobre todo, tan importantes y simbólicos ajuares han dado pie a interpretar el edificio como lugar de culto (Belén 2000) y a vincularlo a una posible comunidad de colonos orientales y/o sus descendientes, grupo que habría ocupado durante el Hierro Antiguo la pequeña meseta de Carmona donde se ubica el barrio de San Blas.

Nuestro interés por analizar las posibles implicaciones astronómicas de esta estructura radica en su identificación previa como centro ceremonial de tipo religioso. No obstante, los trabajos arqueológicos no hallaron en ninguna fase del edificio los altares que habrían certificado esa función comúnmente aceptada.

Como el solar donde apareció el edificio de Saltillo, en el caso histórico de Carmona, se encuentra hoy ocupado por viviendas, nuestro trabajo de campo se tuvo que limitar a medir los acimutes de las paredes de la manzana moderna que se incluyen en la planimetría del yacimiento. Con estos datos hemos estimado la orientación de los muros antiguos del 'Ámbito 6' del san-
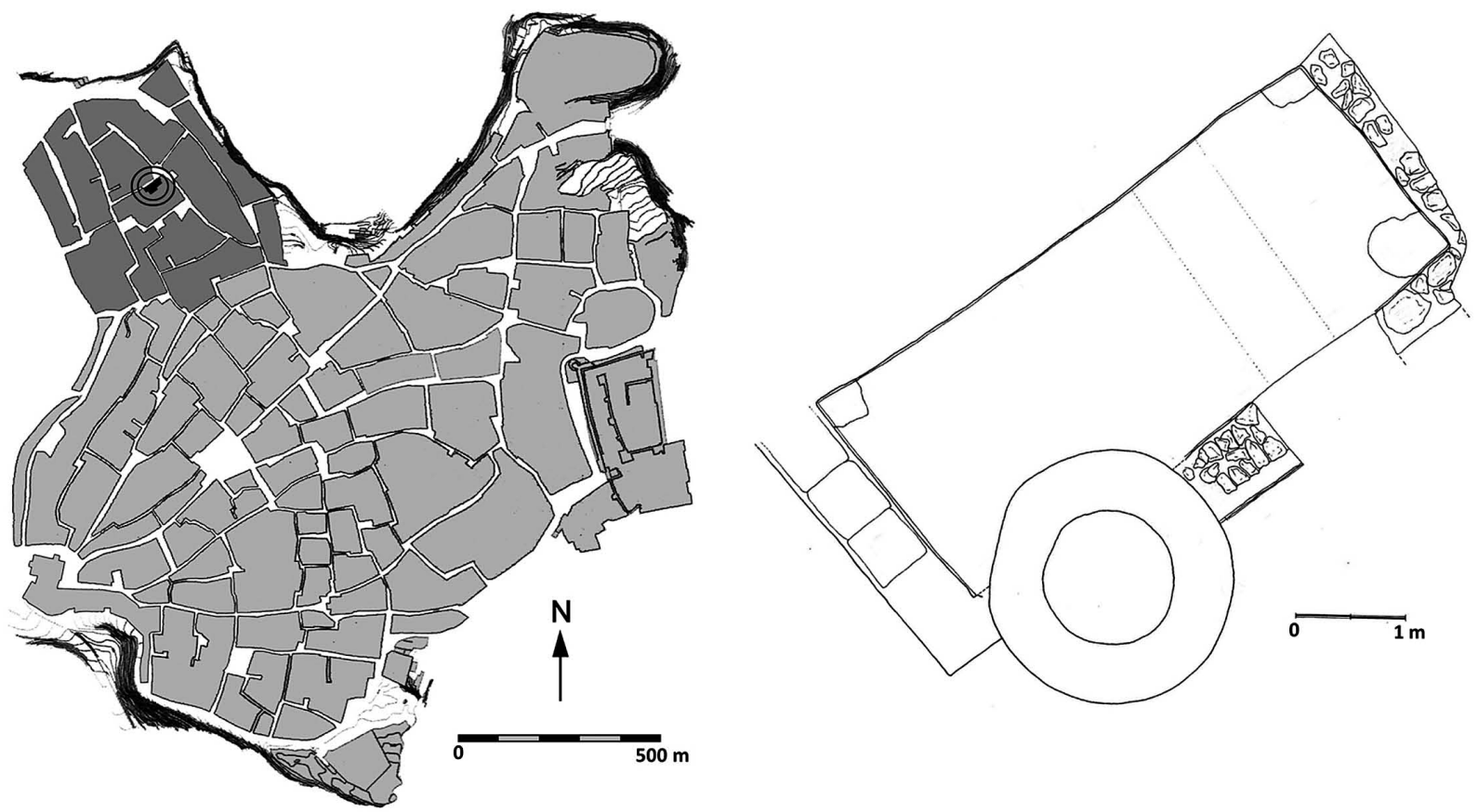

Fig. 4. A la izquierda, ubicación del edificio 'Ámbito 6' del yacimiento tartésico de Saltillo (círculos concéntricos), en el barrio fenicio de Carmona (Sevilla) y a la derecha detalle del mismo (Belén et al. 1997).

Trab. Prehist., 70, N. ${ }^{\circ}$ 1, enero-junio 2013, pp 114-139, ISSN: 0082-5638

doi: $10.3989 /$ tp.2013.12105 
tuario, a partir de los ángulos definidos por estas estructuras y por las actuales (Fig. 4). La declinación magnética utilizada para transformar ángulos horizontales medidos con la brújula a acimutes fue la misma que la obtenida horas antes en la Necrópolis Romana de Carmona a partir de observaciones solares con el teodolito (2). Debido a las características geológicas del lugar, no esperamos variaciones locales del campo magnético, suponiendo la misma declinación magnética en ambos yacimientos. De hecho, las declinaciones magnéticas que hemos determinado para los distintos sitios de época tartésica estudiados en el presente trabajo son casi coincidentes (la dispersión es solo del orden de $0,5^{\circ}$ ), lo que refrenda nuestra suposición.

En su momento el trazado de la sala del santuario de Saltillo descrita más arriba no coincidía con el de las construcciones protohistóricas superpuestas. La orientación del eje mayor del habitáculo presenta un acimut de $53,5^{\circ} \pm 2,0^{\circ}$, similar al del altar de Coria del Río y al de la estancia A-40 del Carambolo (capilla del altar taurodérmico). Si tomamos, también en este caso, la orientación hacia oriente como la significativa y un horizonte de $h=0^{\circ}$ (es imposible medirlo debido a que se encuentra en el interior de la ciudad de Carmona), obtenemos una declinación de $+27,8^{\circ} \pm 1,5^{\circ}$.

\subsection{La Mesa de Setefilla (Lora del Río, Sevilla)}

Este complejo yacimiento arqueológico está ubicado en la vertiente meridional de Sierra Morena, al pie del arroyo Guadalbacar, subsidiario del Guadalquivir por la derecha. El asentamiento, prehistórico, protohistórico, romano y medieval, ocupa una plataforma rocosa que domina una significativa vía de comunicaciones que une el valle bético con la Meseta central española. Al pie del asentamiento se ubica una importante necrópolis de tiempos tartésicos y romanos conocida en la literatura arqueológica al menos desde el primer tercio del siglo XX (Bonsor y Thouvenot 1928;

(2) Aprovechando nuestra visita a Carmona, medimos también las orientaciones de algunas tumbas romanas de su necrópolis, aunque estos datos no forman parte del presente artículo.
Aubet 1975, 1978). El área más destacable en este trabajo es un potente tell ubicado al norte de la actual ermita de la Virgen de Setefilla y bajo el castillo de época islámica. M. E. Aubet ha dirigido aquí diversos trabajos, de los cuales nos importa ahora solo el sondeo conocido como 'Corte 3' (Aubet et al. 1983).

Los dos edificios del Hierro Antiguo analizados por nosotros se emplazaron por tanto sobre una pequeña acrópolis, entonces amurallada, de unos $5000 \mathrm{~m}^{2}$ de extensión. Sobre esa base y las importantes medidas de sus paredes, cabe pensar que formaran parte de estructuras de considerable envergadura e importancia dentro del asentamiento (Fig. 5). Si se unen tales características a su fabricación, en un caso con grandes sillares calizos trabados en cremallera (Aubet et al. 1983: fig. 9 y láms. III-IV), se percibe de inmediato la relevancia que estas construcciones debieron tener en su día. Tales hechos han llevado a que, al menos el edificio de sillares, se considere parte de una singular construcción pública de tipo religioso o social (Aubet et al. 1983: 37-38). Desconocemos su respectiva función, pero tal vez podrían aclarar los papeles concretos de cada conjunto algunos hallazgos producidos con posterioridad a las excavaciones de los años 1970, que incluyen algunos exvotos del Hierro Antiguo y que podrían por tanto señalar al mundo de las creencias (Ladrón de Guevara et al. 1992). Pero como tales datos nuevos proceden de unas obras no controladas arqueológicamente y de un sector del yacimiento relativamente alejado de los complejos arquitectónicos que ahora nos interesan, su valor científico es siempre relativo.

Tras su descubrimiento en 1979, y para garantizar su protección como en los casos señalados de Coria del Río y del Carambolo, los muros aparecidos en el Corte 3 fueron sepultados de nuevo con la misma tierra extraída durante la excavación arqueológica. Ello nos impidió medir de forma directa la orientación de estas estructuras en la visita que hicimos al yacimiento en abril de 2006. Por ese motivo, nuestro estudio consistió en anotar la orientación de varios lienzos de muralla y de algunas paredes de la torre del homenaje de la fortaleza medieval. A partir de estos datos hemos estimado la disposición astronómica de las estructuras arqueológicas que nos interesan sobre la planimetría disponible del yacimiento, espe- 

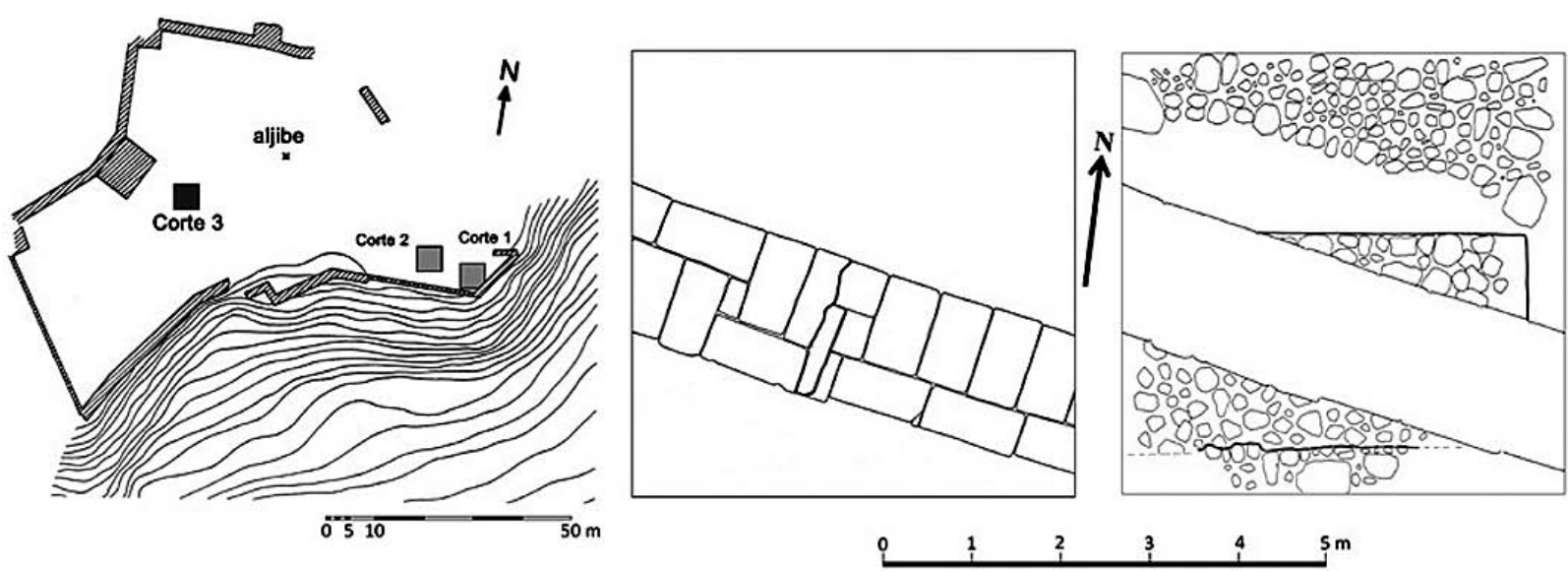

Fig. 5. A la izquierda, plano general del sector norte de la Mesa de Setefilla (Lora del Río, Sevilla). Las estructuras estudiadas se ubican en el Corte 3. Los planos corresponden a los estratos de época tartésica VI (centro) y VII (derecha) de las excavaciones realizadas por Aubet et al. (1983). Se midió la orientación de la estructura de sillares del estrato VI y la del muro de mampostería irregular del estrato VII (marcada con trazo más oscuro).

cialmente las plantas publicadas en la memoria de excavación (Aubet et al. 1983: 37-39, figs. 9 y 10).

La alineación de los dos muros estudiados, el de sillares y el de mampostería que corre inmediatamente debajo de este, es ligeramente distinta. La estructura superior o edificio de sillares, que pertenece al estrato VI y que puede fecharse en los momentos finales del Hierro Antiguo, presenta un acimut de $100,5^{\circ}$ (hacia el este), que correspondería al orto de un astro con declinación $-8^{\circ}$ (suponiendo un horizonte con $h=0^{\circ}$ ). El muro del estrato VII, más antiguo y más ancho, y construido con mampostería irregular, muestra un acimut de $83^{\circ}\left(\right.$ declinación $\left.=+5,5^{\circ}\right)$. La excavación de 1979, la que exhumó estas construcciones, no proporcionó datos sobre la ubicación de las respectivas puertas de los edificios a los que pertenecieron en su día tales muros. Por ello hemos supuesto, esta vez solo de forma hipotética, que la dirección relevante fue la oriental como en los demás casos analizados.

\subsection{El 'edificio público' de Tejada la Vieja (Escacena del Campo, Huelva)}

En Tejada la Vieja se ubican los restos de una gran ciudad fortificada de época tartésica que vi- vió básicamente de la minería y metalurgia de la plata (Fernández Jurado 1987; Fernández Jurado y García Sanz 2001) (Fig. 6). El yacimiento con 6,4 ha de extensión está emplazado sobre una meseta compuesta por tres terrazas escalonadas. La muralla se extiende a lo largo de $1474 \mathrm{~m}$ y solo conserva su base pétrea, un glacis característico de las defensas urbanas de las ciudades siropalestinas. Este tipo de fortificación llegó precisamente a la Península Ibérica en esa época de manos de la diáspora fenicia (Escacena 2005: 198-205). Los primeros estudios científicos hacen referencia a una ocupación de la Edad del Bronce (Blanco y Rothenberg 1981: 281). Sin embargo las excavaciones llevadas a cabo por el Servicio de Arqueología de la Diputación de Huelva sugieren una fundación no anterior al siglo VIII a.C., correspondiente a las primeras construcciones de edificios públicos y de viviendas, así como a los momentos iniciales de la fortificación que rodea todo el asentamiento. La ocupación humana permanente duró hasta el siglo IV a.C., si bien se perciben cambios sustanciales en el siglo VI a.C., cuando su economía pasa a ser fundamentalmente agraria y ganadera. Por no pervivir hasta época romana, se desconoce su nombre antiguo. Muchos investigadores interpretan el yacimiento de Tejada la Vieja como los restos de una fundación tartésica, dándole a este calificativo valor étnico además de cronológico. De ser así, no se trataría 

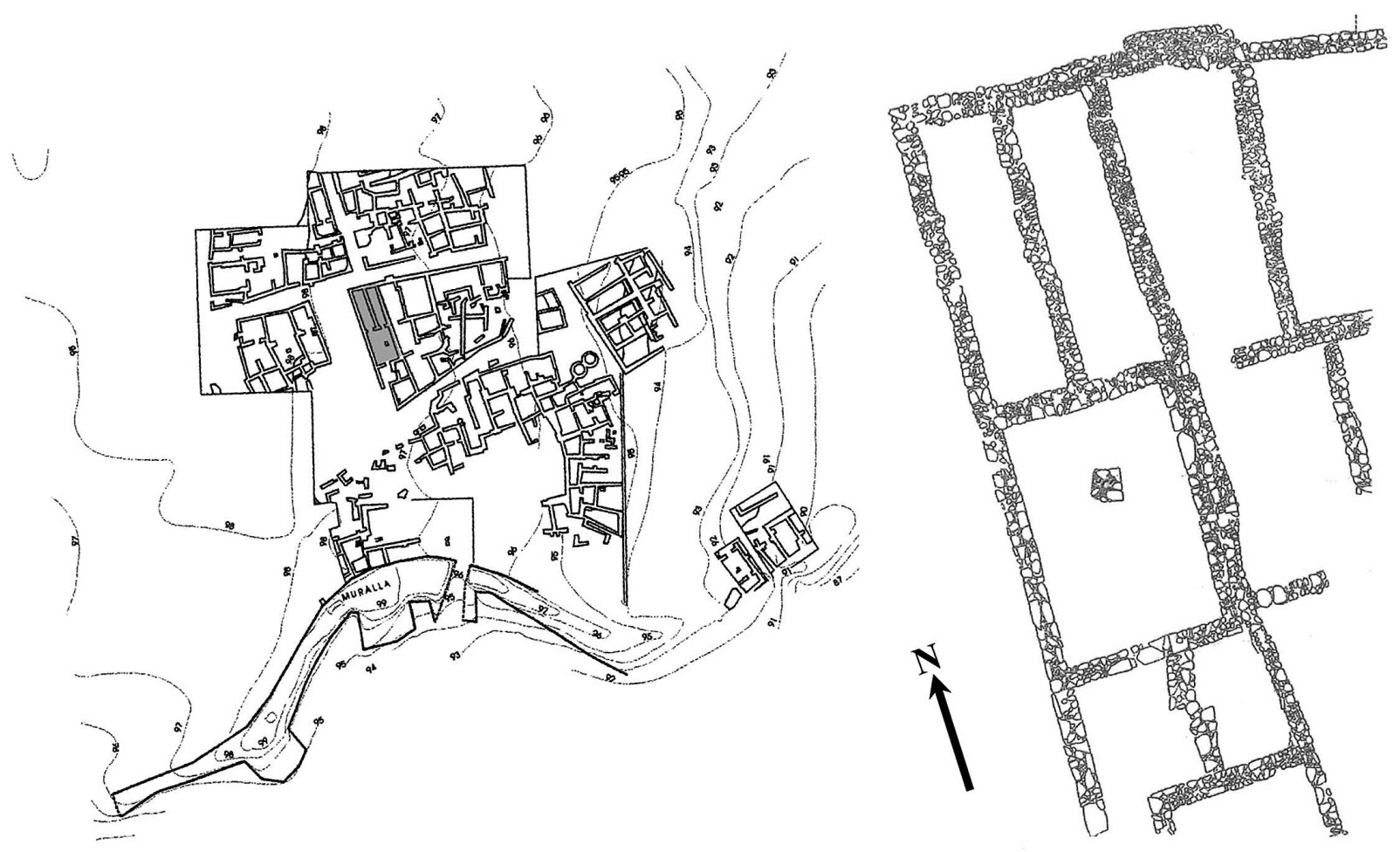

Fig. 6 Plano general de la acrópolis del poblado de Tejada la Vieja (Escacena del Campo, Huelva) a la izquierda. El área gris corresponde al edificio público de época turdetana ampliado en el plano de la derecha y cuya orientación se ha estudiado. Esta figura se basa en la planimetría facilitada por J. Fernández Jurado (Diputación Provincial de Huelva).

de un enclave fenicio. Pero la ausencia de un hábitat anterior a los momentos de la colonización oriental sugiere la implicación de la población cananea en su nacimiento. Se desconoce aún la ubicación de $\mathrm{su}(\mathrm{s})$ necrópolis.

Aquí el estudio arqueoastronómico de campo se centró en la medida de la orientación de las paredes de varias estancias de una estructura, al parecer de uso público, situada en la parte alta de la población (terraza superior) y que se distribuye entre los cuadros A-10 y A-6 excavados en la campaña de 1986 (Fernández Jurado 1987: 77-82 y figs. 22 y 23). Es un edificio tenido en ocasiones por 'singular' y que corresponde a la fase III del hábitat, fechada desde mediados del siglo VI hasta mediados del IV a.C. En el plano de la superficie excavada de la ciudad publicado por Fernández Jurado (1987: 91), reproducido parcialmente en nuestra figura 6, vemos mayor grado de regularidad en este complejo arquitectónico (paralelismo entre las paredes enfrentadas y perpendicularidad de los muros en las esquinas) que en las demás construcciones exhumadas, que también se distribuyen de forma más aleatoria por este sector del hábitat. Hacia el oeste linda con una calle de unos $7 \mathrm{~m}$ de anchura. Cabe señalar que las paredes de este edificio medidas por nosotros y el resto de estancias que se encuentran en la actualidad en superficie en esta zona de la acrópolis corresponden a la época final de la ciudad, ya del siglo IV a.C. Debido a esta cronología se ha dado siempre este momento por turdetano (Hierro Reciente). Se trata por tanto de una construcción más tardía que el resto de las que estudiamos.

Las medidas de la orientación de los distintos muros se obtuvieron con la brújula de precisión, mientras que la declinación magnética se determinó a partir de observaciones de la posición del Sol con el teodolito. Del resultado de tales cómputos se puede deducir que el edificio público aludido se orienta de una forma muy precisa con relación a los ejes determinados por los puntos cardinales. Esto contrasta ya con el resto de las estructuras aquí estudiadas, que pertenecen al Hierro Antiguo. 
La única entrada aparente al complejo está en su fachada sur. Desde aquí se accedería a un espacio subdividido en dos pequeñas estancias, y más adelante a un patio interior. El fondo aparece de nuevo compartimentado, en este caso en dos habitaciones alargadas y paralelas entre sí, de similar diseño y extensión, con accesos independientes desde el patio. Considerando la dirección meridional como la relevante, ya que hacia ella abren los vanos del edificio, encontramos que el eje mayor de la estructura apunta a un acimut de $179^{\circ} \pm 2^{\circ}$, justamente hacia el sur geográfico (definido por la dispersión de los acimutes medidos para las distintas paredes). Esta estructura, que se ha considerado de posible uso público, es de gran regularidad y mayor calidad en relación con las del entorno inmediato. Este rasgo, unido a su orientación, sugiere que su localización en el espacio fue un elemento determinante en su diseño y construcción, lo que refuerza su carácter de edificio singular.

\section{EL ORAL (SAN FULGENCIO, ALICANTE): LA HABITACIÓN IIIJ1}

El Oral es uno de los asentamientos prerromanos más antiguos del sureste peninsular ibérico, pues se inicia en la primera mitad del siglo $\mathrm{V}$ a.C. (Abad y Sala 1993: 239). Sin embargo ese comienzo del hábitat es más tardío que el de la mayoría de los analizados en el apartado 3. Decidimos incluirlo por contar con un posible altar en forma de piel de toro similar al de Caura y, sobre todo, al del Carambolo. Como este último, el altar de El Oral es mas bien una impronta elaborada sobre el suelo de la denominada habitación IIIJ1, ámbito que se interpreta como espacio sacro (Abad y Sala 1993: 80). Numerosos aspectos arquitectónicos del yacimiento resultan también del mayor interés por sus vínculos estrechos con tradiciones edilicias mediterráneas, que pueden ponerse en relación directa con el mundo fenicio y púnico (Sala y Abad 2006: 25). Son características presentes también en el mundo tartésico, donde las innovaciones del Hierro Antiguo, especialmente las referidas a la forma, disposición interna y orientación de las estructuras y del urbanismo, parecen romper con la herencia supuestamente indígena (Izquierdo 1998). Como en el Carambolo, ese fuerte componente semita se observa en el uso frecuente de pavimentos de conchas como elementos mágicos protectores de determinados espacios (Escacena y Vázquez 2009: 46-47).

La visita al yacimiento se llevó a cabo en abril de 2010 con posterioridad a la de los enclaves andaluces. Se tomaron medidas de la orientación de las paredes de la estancia IIIJ1 con la brújula de precisión, empleando el teodolito en el análisis astronómico del horizonte visible desde el poblado. La declinación magnética para la corrección de las medidas obtenidas con la brújula se determinó a partir de observaciones de la posición del Sol y de cotas topográficas tomadas con ambos instrumentos.

La habitación IIIJ1 se abre a la calle III-IV por medio de un acceso situado en su ángulo sureste, pero dispone de otra entrada en el ángulo opuesto, el noroeste. Aquí se conserva un umbral de piedras planas que denota claramente un vano que comunica con el espacio abierto III.I (Fig. 7). Las paredes septentrional y meridional de este pequeño recinto son perfectamente paralelas dentro de la incertidumbre de $1^{\circ}$ de las medidas, siendo el muro occidental perpendicular a los anteriores. Considerando de nuevo la dirección oriental como la relevante, el eje mayor del edificio apunta hacia un acimut de $54,4^{\circ} \pm 1,0^{\circ}$. Teniendo en cuenta la altura actual del horizonte en esa dirección, que no corresponde con el mar sino con una loma cercana de una altitud aproximada a la del poblado, la declinación del astro que tiene su orto por ese punto es de $+27,2^{\circ} \pm 0,6^{\circ}$.

El hipotético altar taurodérmico, cuya función como tal ara podría suscitar dudas al carecer de focus, no era ya visible en el momento de la visita. La definición del acimut se estimó por su eje mayor a partir del plano detallado publicado por Abad y Sala (1993: 152), reproducido en nuestra figura 7, y de las medidas de la orientación de las paredes de la habitación que lo albergaba, la IIIJ1. Suponiendo de nuevo la dirección este como la significativa, puede definirse un acimut aproximado de $65,5^{\circ}$. Es de destacar que el eje mayor del supuesto altar, definido primeramente como "emblema" por los excavadores (Abad y Sala 1993: 91-93), apunta, a través de la entrada de la habitación, hacia la línea del horizonte marino, a un punto concreto donde tiene su orto un astro con declinación de unos $+18,7^{\circ}$. 


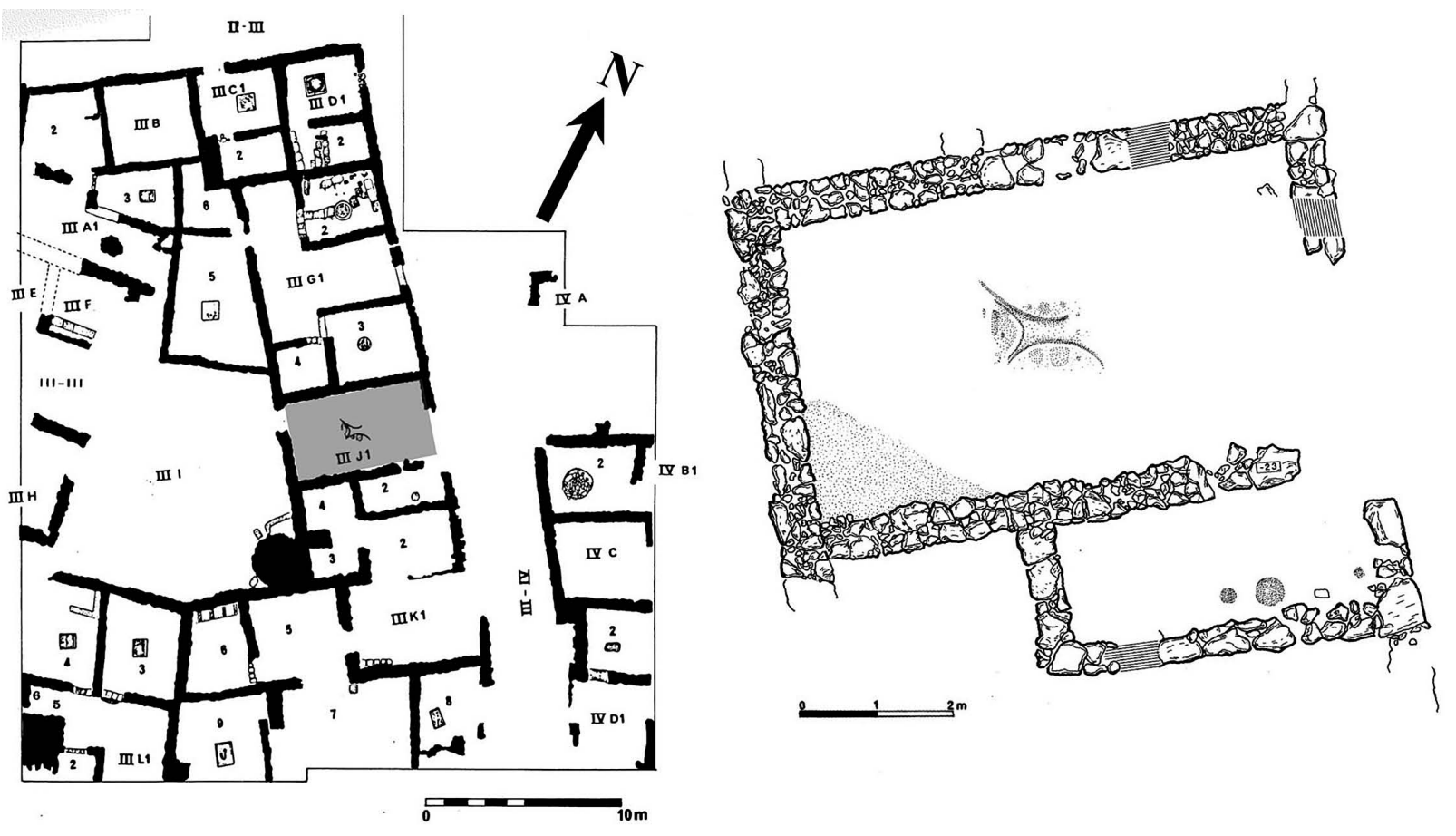

Fig. 7. Plano general del sector III del poblado ibérico del Oral (San Fulgencio, Alicante) a la izquierda. La casa IIIJ1, sombreada, aparece a la derecha, en un plano detallado con el altar en su centro (según Abad y Sala 1993: figs. 40 y 139 respectivamente adaptadas).

El análisis del horizonte visible desde el Oral proporcionó resultados interesantes en la dirección occidental. Los puntos del horizonte oriental hacia donde enfocan el eje mayor de la estancia IIIJ1 y el altar no presentan ninguna particularidad. El primero se orienta hacia una pequeña elevación de perfil muy suave localizada entre la costa y el poblado, a unos escasos centenares de metros, y el segundo hacia el horizonte marino. Sin embargo, hacia el oeste las paredes de la estancia IIIJ1 apuntan exactamente hacia un elemento topográfico bastante llamativo del horizonte lejano (Fig. 8): la cumbre de la Sierra de Pujálvarez, situada a unos $30 \mathrm{~km}$ entre las provincias de Alicante y Murcia. Otro resultado sugerente es que el ocaso solar durante los equinoccios, visto desde el poblado, se produce sobre el perfil de la Sierra de Callosa, cerca de la cumbre. Este conjunto montañoso, situado a unos $20 \mathrm{~km}$ de distancia, es el elemento topográfico de mayor altura de todo el horizonte lejano que rodea al yacimiento. En esta dirección hay montículos de altura similar, pero mucho más cercanos. Son producto de la remoción reciente de tierra con el fin de urbanizar los alrededores lo que, desgraciadamente, ha destruido parte del área occidental del poblado (Fig. 9).

\section{ANÁLISIS CRÍTICO DE LOS RESULTADOS}

En la tabla 1 resumimos los resultados de las secciones 3 y 4 . Solo se han tenido en cuenta eventos astronómicos relacionados con el Sol, la Luna o Venus, los cuerpos celestes visibles a ojo desnudo más brillantes y que a priori podrían tener una mayor importancia simbólica. No podemos distinguir de forma clara en el resto de planetas que describen órbitas poco inclinadas respecto a la de la Tierra si una orientación está dirigida realmente a cualquiera de ellos o de los tres astros ya citados. Tampoco consideramos orientaciones hacia estrellas debido a que son multitud las que podemos ajustar a la orientación medida en el intervalo de tiempo en que se usó 


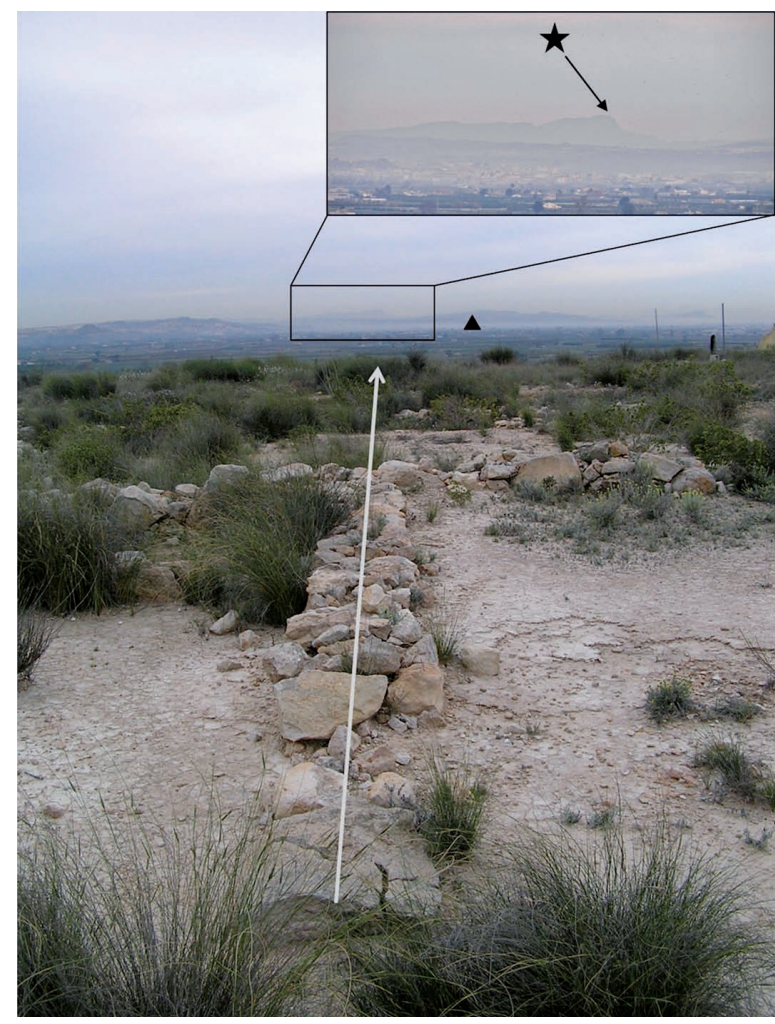

Fig. 8. Vista parcial del horizonte suroeste desde la casa IIIJ1 del poblado ibérico del Oral (San Fulgencio, Alicante). La flecha blanca señala la dirección hacia la que apunta el muro meridional de la casa (de unos $7 \mathrm{~m}$ de longitud): la posición del pico de la Sierra de Pujálvarez, uno de los elementos más lejanos del horizonte visible desde el yacimiento. Justo allí se produce la puesta de Venus en su momento de declinación máxima sur como estrella de la tarde o vespertina (DMSVV, correspondiente a una declinación de $-26,5^{\circ}$ ). Es el punto más al sur por dónde ver al planeta Venus ponerse a lo largo de su ciclo de 8 años. La foto de la esquina superior derecha amplía la zona del pico. La flecha negra indica la trayectoria que sigue Venus justo antes de su ocaso. El triángulo negro sobre el horizonte a la derecha de la Sierra de Pujálvarez señala el punto donde se produce el ocaso del Sol en el solsticio de invierno (PSI, la posición solar más meridional, con declinación $\left.=-23,7^{\circ}\right)$.

el edificio (3). Como la altura sobre el horizonte a la que comienza a ser visible (o termina de verse) una estrella después de su orto (o antes de

(3) La posición de las estrellas sobre el fondo celeste cambia con el tiempo debido a la precesión de los equinoccios (movimiento relativo de los planos ecuatorial y orbital terrestres), lo que no afecta a las posiciones del Sol, la Luna o los planetas del Sistema Solar.

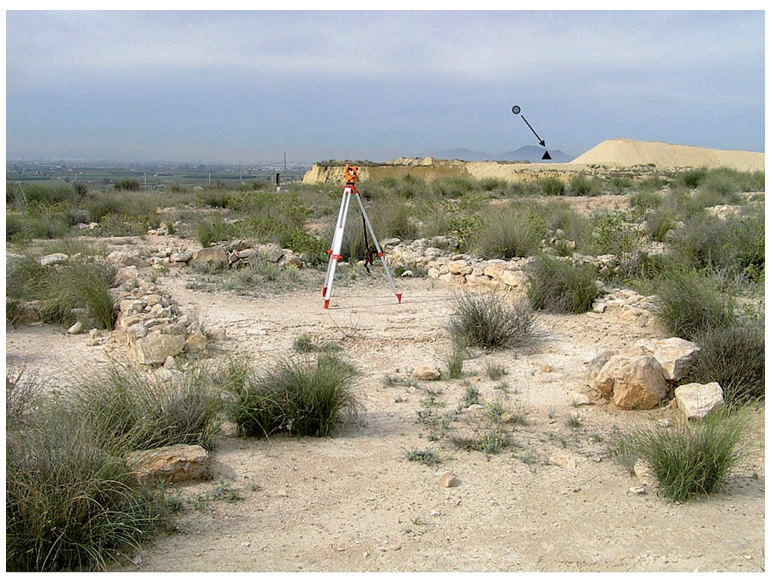

Fig. 9. Vista parcial del horizonte occidental desde la casa IIIJ1 del poblado ibérico del Oral (San Fulgencio, Alicante). Los muros en torno al teodolito corresponden a la casa y están separados unos cuatro metros. El triángulo negro indica el punto del horizonte donde se produce el ocaso del Sol en los equinoccios, muy cerca de la cumbre de la Sierra de Callosa, el punto más alto del horizonte lejano visible desde el yacimiento. Los amontonamientos de tierra amarilla situados delante son producto de las actividades de extracción de áridos que han alterado la topografía de Sierra del Molar en esa dirección. Desgraciadamente, desconocemos el perfil original de la Sierra de Callosa en época ibérica, por lo que no podemos saber con seguridad si era visible desde el yacimiento. La flecha negra indica la trayectoria que sigue el Sol justo antes de su ocaso. El pequeño círculo reproduce el tamaño aparente del disco solar.

su ocaso) depende fuertemente de su brillo y de las condiciones atmosféricas en el momento de la observación, por lo general, una orientación hacia la salida o puesta de una estrella no puede establecerse con precisión.

Una inspección rápida a los resultados de la tabla 1 parece mostrar una gran cantidad de relaciones astronómicas en los santuarios estudiados. Ello se debe a que las orientaciones de muchos de los elementos medidos entran en la franja donde podemos encontrar ortos u ocasos solares, lunares y venusinos en posiciones extremas de su movimiento orbital sobre la esfera celeste. El resultado más llamativo es que la orientación de elementos relevantes de cuatro de las estructuras arquitectónicas (el Santuario I y el altar del Santuario III de Caura, la estancia A-40 del Carambolo o capilla sur de las fases Carambolo IV y III, la habitación de Saltillo en Carmona y la casa IIIJ1 del Oral) es idéntica dentro de los errores aceptables de las 


\begin{tabular}{|c|c|c|c|c|c|c|c|}
\hline Santuario & $\begin{array}{c}\text { Cronología } \\
\text { Época cultural }\end{array}$ & $\begin{array}{c}\text { Elemento } \\
\text { arquitectónico }\end{array}$ & $\begin{array}{l}\text { Dirección } \\
\text { relevante }\end{array}$ & $\underset{\left({ }^{\circ}\right)}{\text { Acimut }}$ & $\begin{array}{c}\text { Altura } \\
\left({ }^{\circ}\right)\end{array}$ & $\begin{array}{c}\text { Declinación } \\
\left({ }^{\circ}\right)\end{array}$ & $\begin{array}{c}\text { Evento } \\
\text { astronómico }^{\mathrm{a}}\end{array}$ \\
\hline \multirow[t]{4}{*}{ Coria } & \multirow{4}{*}{ Orientalizante } & \multirow[t]{2}{*}{$\begin{array}{l}\text { Altar (fase III) y } \\
\text { edificio fase } \mathrm{I}^{\mathrm{b}}\end{array}$} & Este & $55,5 \pm 2,0$ & 0 & $+26,4 \pm 1,5$ & $\begin{array}{c}\text { ¿SLMN, } \\
\text { DMNVM? }\end{array}$ \\
\hline & & & Oeste & $235,5 \pm 2,0$ & 0 & $-27,2 \pm 1,5$ & DMSVV \\
\hline & & \multirow[t]{2}{*}{ Edificio fase $\mathrm{IV}^{\mathrm{b}}$} & Este & $62,0 \pm 2,5$ & 0 & $+21,6 \pm 2,0$ & ¿SSV? \\
\hline & & & Oeste & $242,0 \pm 2,5$ & 0 & $-22,3 \pm 2,0$ & PSI \\
\hline \multirow[t]{11}{*}{ Carambolo } & \multirow{11}{*}{$\begin{array}{c}\text { Fin IX a.C.- fin VII a.C. } \\
\text { Orientalizante }\end{array}$} & \multirow[t]{3}{*}{ Patio A-29 } & \multirow[t]{2}{*}{ Este } & \multirow[t]{2}{*}{$55,8 \pm 1,0$} & 0 & $+26,2 \pm 1,0$ & \\
\hline & & & & & 2 & $+27,7 \pm 1,7$ & SLMN \\
\hline & & & Oeste & $235,8 \pm 1,0$ & 0 & $-26,9 \pm 1,0$ & DMSVV \\
\hline & & \multirow{3}{*}{$\begin{array}{c}\text { Estancia A-46 } \\
\text { Carambolo V }\end{array}$} & \multirow[t]{2}{*}{ Este } & \multirow[t]{2}{*}{$58,8 \pm 1,0$} & 0 & $+24,0 \pm 1,0$ & SSV, DMNVM \\
\hline & & & & & 2 & $+25,5 \pm 1,7$ & $\begin{array}{c}\text { ¿SSV? } \\
\text { DMNVM }\end{array}$ \\
\hline & & & Oeste & $238,8 \pm 1,0$ & 0 & $-24,7 \pm 1,0$ & PSI \\
\hline & & \multirow{3}{*}{$\begin{array}{l}\text { Estancia A-40 } \\
\text { Carambolo IV-III } \\
\text { Templo sur }\end{array}$} & \multirow[t]{2}{*}{ Este } & \multirow[t]{2}{*}{$54,6 \pm 2,0$} & 0 & $+27,0 \pm 1,5$ & SLMN \\
\hline & & & & & 2 & $+28,5 \pm 2,2$ & SLMN \\
\hline & & & Oeste & $234,6 \pm 2,0$ & 0 & $-27,7 \pm 1,5$ & $\begin{array}{l}\text { DMSVV, } \\
\text { ¿PLMS? }\end{array}$ \\
\hline & & \multirow{2}{*}{$\begin{array}{l}\text { Estancia A-1 } \\
\text { Carambolo IV-III }\end{array}$} & \multirow[t]{2}{*}{ Este } & \multirow[t]{2}{*}{$63,5 \pm 2,0$} & 0 & $+20,4 \pm 1,5$ & \\
\hline & & & & & 2 & $+21,8 \pm 2,2$ & SSV \\
\hline \multirow{4}{*}{ Saltillo } & \multirow{4}{*}{$\begin{array}{c}\text { Fin VII a.C. - V a.C. } \\
\text { Orientalizante }\end{array}$} & \multirow{4}{*}{$\begin{array}{l}\text { Templo norte } \\
\text { Habitación }{ }^{\mathrm{b}}\end{array}$} & Oeste & $243,5 \pm 2,0$ & 0 & $-21,1 \pm 1,5$ & \\
\hline & & & Este & $53,5 \pm 2,0$ & 0 & $+27,8 \pm 1,5$ & SLMN \\
\hline & & & Oeste & $233,5 \pm 2,0$ & 0 & $-28,5 \pm 1,5$ & $\begin{array}{c}\text { PLMS, } \\
\text { ¿DMSVV? }\end{array}$ \\
\hline & & & Sur & $143,5 \pm 2,0$ & 0 & $-40,0 \pm 1,5$ & \\
\hline \multirow[t]{4}{*}{ Setefilla } & \multirow{4}{*}{$\begin{array}{c}\text { Fin VIII a.C.- mitad VI a.C. } \\
\text { Orientalizante }\end{array}$} & \multirow[t]{2}{*}{ Muro estrato $\mathrm{VI}^{\mathrm{b}}$} & Este & $100,5 \pm 2,0$ & 0 & $-8,6 \pm 1,5$ & \\
\hline & & & Oeste & $280,5 \pm 2,0$ & 0 & $+8,0 \pm 1,5$ & \\
\hline & & \multirow[t]{2}{*}{ Muro estrato VII ${ }^{\mathrm{b}}$} & Este & $83,0 \pm 2,0$ & 0 & $+5,2 \pm 1,5$ & \\
\hline & & & Oeste & $263,0 \pm 2,0$ & 0 & $-5,9 \pm 1,5$ & \\
\hline \multirow{2}{*}{$\begin{array}{c}\text { Tejada la } \\
\text { Vieja } \\
\end{array}$} & \multirow{2}{*}{\begin{tabular}{|c|} 
Mitad VI a.C. $-\operatorname{mitad}$ IV \\
a.C. \\
Turdetana \\
\end{tabular}} & \multirow[t]{2}{*}{ Edificio público } & Sur & $179 \pm 2$ & 0 & $-53,0 \pm 1,5$ & $\begin{array}{c}\text { Punto cardinal } \\
\text { Sur } \\
\end{array}$ \\
\hline & & & Este & $89 \pm 2$ & 0 & $+0,5 \pm 1,5$ & SE \\
\hline \multirow[t]{4}{*}{ El Oral } & \multirow{4}{*}{$\begin{array}{l}\text { Primera mitad V a.C. } \\
\text { Ibérica }\end{array}$} & Casa IIIJ1 & Este & $54,4 \pm 1,0$ & 0,4 & $+27,2 \pm 0,6$ & ¿SLMN? \\
\hline & & & Oeste & $234,4 \pm 1,0$ & 0,7 & $-27,1 \pm 0,6$ & DMSVV \\
\hline & & Altar $^{\mathrm{b}}$ & Este & $65,5 \pm 2,0$ & 0 & \begin{tabular}{|l|l}
$+18,7 \pm 1,5$ \\
\end{tabular} & \\
\hline & & & Oeste & $245,5 \pm 2,0$ & 0,5 & $-19,0 \pm 1,5$ & \\
\hline
\end{tabular}

Tab. 1. Orientaciones astronómicas en edificios protohistóricos del sur de la Península Ibérica (localizados en figura 1). La dirección es la de orientación del elemento (hemisferio este $u$ oeste del horizonte, es decir, ortos u ocasos de astros). La altura es la del punto en que intersecta el acimut con el horizonte. La declinación celeste corresponde al par acimut-altura y al evento astronómico relacionable con dicha orientación. Un evento astronómico se asigna a una orientación dada cuando su declinación coincide con el margen de error de la medida.

${ }^{a}$ Entre interrogantes los marginalmente consistentes con la declinación asociada con la orientación (incrementando el error en $\left.0^{\circ}, 5\right)$.

SSV - salida del Sol en el solsticio de verano (declinación $\left.=+23^{\circ}, 7\right)$.

PSI - puesta del Sol en el solsticio de invierno (declinación $=-23^{\circ}, 7$ ).

$\mathrm{SE}$ - salida del Sol en equinoccios (declinación $=0^{\circ}$ ).

$\mathrm{PE}-$ puesta del Sol en equinoccios (declinación $=0^{\circ}$ ).

SLMN - salida de la Luna en el lunasticio mayor norte (declinación $\left.=+28^{\circ}, 2\right)$.

PLMS - puesta de la Luna en el lunasticio mayor sur (declinación $\left.=-29^{\circ}, 7\right)$.

DMNVM - declinación máxima norte de Venus como estrella matutina (visible al este antes del orto solar, declinación = +24²,6).

DMSVV - declinación máxima sur de Venus como estrella vespertina (visible al oeste tras el ocaso solar, declinación $=-26^{\circ}, 5$ ).

${ }^{\mathrm{b}}$ Medidas tomadas sobre plano. 
medidas tomadas con un acimut representativo de alrededor de $55^{\circ}$. El hallazgo parece indicar la existencia de un patrón de orientación bien definido en todos estos complejos arquitectónicos. Dicha regularidad reforzaría el carácter sagrado de esas construcciones, establecido en cualquier caso por otras vías.

En los momentos iniciales de nuestro estudio intentamos relacionar las orientaciones encontradas solo con posiciones solares o lunares. La repetición en varios sitios de elementos con un acimut de alrededor de $55^{\circ}$, medida que no coincidía con puntos singulares de la órbita de los dos astros principales como solsticios y lunasticios, nos hizo contar con Venus en el análisis. En atención al nombre usado para algunos otros grupos de orientaciones astronómicas (4), aludiremos a nuestro conjunto con el término de 'familia de $55^{\circ}$. La inclusión de Venus abre el espectro de explicaciones posibles. Indicaremos los argumentos a favor y en contra de todas ellas. Pero, antes de analizar en profundidad la orientación de los santuarios de la 'familia de $55^{\circ}$ ', resumiremos las posibles relaciones astronómicas identificadas en los yacimientos incluidos en este trabajo que no forman parte de dicho lote.

En primer lugar, la orientación de los dos muros de sendas estructuras que se midieron sobre plano en el yacimiento de Setefilla es algo diferente y en ningún caso relacionable con una posición singular de algún astro relevante. Por el contrario, el edificio público de Tejada la Vieja, perteneciente al Hierro II y por tanto posterior a la serie tartésica, muestra una disposición bastante precisa a lo largo de los ejes cardinales, con su entrada apuntando hacia el sur geográfico. Orientaciones en la dirección de los ejes cardinales, y en especial hacia el este, son muy comunes en los santuarios del mundo prerromano del sureste peninsular hispano, donde los equinoccios podrían jugar un importante papel en el ritual (Esteban 2002; Esteban y Moret 2006). Analizaremos ahora en profundidad los resultados relativos a los santuarios de la 'familia de $55^{\circ}$ '.

No se conoce con seguridad dónde se ubicaba el acceso al templo de Coria del Río, pero ya

(4) Se conoce como 'familia de $17^{\circ}$ ' a un grupo numeroso de ciudades y templos prehispánicos mesoamericanos que comparten una orientación común con acimutes entre $15^{\circ}$ y 20 (Aveni 1991: 269). hemos señalado la presencia de un apéndice con marcada significación ritual en el flanco oriental, uno de los lados menores del altar. Este hecho sugirió en su día que esta parte del ara podría estar marcando la dirección relevante (Escacena 2009: 106). Tal elemento alude formalmente al trozo del cuello de la piel de toro imitada por la silueta del altar y que en su receptáculo central se colocaría sangre del animal inmolado (Escacena e Izquierdo 2008: 437). Si nos fijamos en la tabla 1 , hacia el este tanto el acimut del altar como el del santuario más viejo apuntan, aunque con una ligera desviación debida a los márgenes de error de la medida, a un punto del horizonte ubicado al sur de la salida de la Luna en el lunasticio mayor norte (Salida Lunasticio Mayor Norte: SLMN), la posición más septentrional que pudo alcanzar la Luna $\left(+28,2^{\circ}\right)$ en la época de frecuentación del edificio (5). También enfoca al norte del punto por donde tiene su orto el planeta Venus en el momento de alcanzar su declinación máxima norte $\left(+24,6^{\circ}\right)$ como estrella de la mañana (Declinación Máxima Norte Venus Matutino: DMNVM). Por el contrario, si consideramos la dirección oeste como la relevante, la declinación deja de tener relación con la Luna para ajustar de forma más exacta con la posición del ocaso de Venus en su momento de declinación máxima sur como estrella de la tarde o vespertina (Declinación Máxima Sur Venus Vespertino: DMSVV, $\left.-26,5^{\circ}\right)$. Por otra parte, la orientación promedio de las paredes del edificio de la fase IV está desviada unos grados hacia el sur con respecto a la orientación longitudinal del altar y al eje supuestamente relevante del Santuario I. Hacia el este, el edificio IV apuntaría, solo con un pequeño error, hacia la salida del Sol en el solsticio de verano (Salida Solsticio Verano: SSV, su posición más septentrional, con una declinación de $+23,7^{\circ}$ ), mientras que hacia el oeste la correspondencia sería con la puesta del Sol en el solsticio de invierno (Puesta Solsticio Invierno: PSI, la posición solar más meridional, declinación $=-23,7^{\circ}$ ) de forma aún más precisa que en el caso anterior. Como vemos, en el santuario de Caura la

(5) Los lunasticios también se denominan paradas mayores. Son equivalentes a los solsticios solares pero, mientras estos son de periodicidad anual, los lunasticios mayores solo se alcanzan cada 18,6 años, el denominado período de regresión de la línea de los nodos. Puede consultarse una explicación más extensa del fenómeno en Aparicio et al. (2000: 33-34) o en Aveni (1991: 88-89).

Trab. Prehist., 70, N. ${ }^{o}$ 1, enero-junio 2013, pp 114-139, ISSN: 0082-5638

doi: $10.3989 /$ tp.2013.12105 
correspondencia de las orientaciones con eventos astronómicos parece más exacta si consideramos la dirección oeste o poniente como la relevante. Esto puede tener una primera explicación, aunque tal vez no la única, en el hecho de que el templo tuvo siempre más despejado el horizonte occidental que el oriental. De hecho, el santuario lindaba por el este con otras muchas construcciones urbanas que obstaculizaban la observación despejada de la línea del horizonte. Según estos datos, el Santuario I y el altar taurodérmico del Santuario III apuntarían hacia la puesta de Venus como lucero de la tarde justo en el momento que alcanza su posición más meridional sobre la esfera celeste (DMSVV), mientras que la fase IV del edificio lo haría hacia el PSI. Es posible que el Santuario III, el que alberga el altar taurodérmico, enfoque hacia la misma posición astronómica que el IV. Esto se cumpliría si su muro perimetral estuviese en idéntica posición que el del templo que se le superpone. Es decir, si ambas paredes coincidiesen a plomo. Este extremo es más que probable; de lo contrario, al menor desvío el muro del Santuario III habría sido localizado durante la excavación, lo que no ocurrió. Como la pared externa del Santuario IV no se retiró, ahora solo es una suposición que la de la fase III discurre exactamente por debajo. Por tanto, de cumplirse esta condición tendríamos que afirmar para el Santuario III lo ya admitido para el IV, además de explicar las razones por las que el eje longitudinal de su altar no es paralelo al muro del edificio que lo cobija.

Antes de continuar con el análisis de los resultados obtenidos para otros sitios aquí estudiados, es necesario detallar las particularidades del movimiento orbital de Venus en general y de sus puntos de declinación extrema en particular. Ello permitirá comprender mejor algunas de las hipótesis que sometemos a verificación (6). Este planeta describe una vuelta completa alrededor del Sol en algo menos de 225 días (lo que se denomina 'periodo sidéreo'). Lo relevante para un observador terrestre es el intervalo de tiempo en que Venus vuelve a estar en la misma posición

(6) Resumimos a continuación la excelente descripción de Sprajc (1996: 17-29 y 139-153) sobre el movimiento orbital de Venus. Su trabajo trata sobre la relevancia de Venus en el simbolismo de las culturas prehispánicas mesoamericanas, pero muchas de sus explicaciones son enteramente aplicables a nuestro contexto. respecto al Sol sobre la esfera celeste. Dicho intervalo se denomina 'periodo sinódico' y dura casi 584 días. Por tanto, el 'periodo sinódico' de Venus es más largo que el 'sidéreo', debido a las diferencias entre los movimientos orbitales de Venus y la Tierra. Así, aunque la Tierra gira alrededor del Sol en el mismo sentido que Venus, lo hace a una menor velocidad angular. Como el periodo sinódico venusino no es un número entero de veces nuestro año terrestre de 365 días, ocurrirá que, después de un periodo sinódico, cualquier posición de Venus en relación con el Sol se volverá a repetir en distinta fecha del año. Podemos comprobar que 5 periodos sinódicos de Venus (unos 2920 días) equivalen casi exactamente a 8 años terrestres, por lo que las mismas posiciones relativas entre el Sol y Venus sobre la esfera celeste se repetirán cada 8 años en las mismas fechas aproximadamente. Además, como Venus orbita a una distancia respecto del Sol menor que la de la Tierra siempre veremos a este planeta relativamente cerca del Astro Rey, poniéndose poco después del ocaso solar (por lo que se le conoce como 'estrella de la tarde') o saliendo poco antes del amanecer (en calidad ahora de 'estrella de la mañana'). Al igual que ocurre con el Sol, los puntos de salida y de puesta de Venus se desplazan a lo largo de los horizontes oriental y occidental respectivamente, llegando en cada uno a alcanzar posiciones extremas hacia el norte y el sur. El valor de las declinaciones máximas que alcanza Venus hacia ambos extremos norte-sur en cada periodo sinódico es variable por tanto en un ciclo de 8 años, aunque también estos patrones de 8 años cambian ligeramente con el tiempo con una oscilación que se repite cada 251 años. Los valores de las declinaciones extremas de Venus son algo mayores que los alcanzados por el Sol en los solsticios debido a que la órbita de este planeta está inclinada algo más de $3^{\circ}$ respecto al plano orbital de la Tierra (plano de la eclíptica). En cualquier caso, las posiciones extremas solares $\mathrm{y}$ venusinas en sus respectivos puntos de orto y ocaso sobre la línea del horizonte nunca estarán alejadas más que unos pocos grados entre sí.

Las declinaciones máximas observables de Venus (tanto norte como sur) sobre los horizontes matutino y vespertino dependen de si estas se producen cuando el planeta es visible como lucero del alba o de la tarde respectivamente. Su valor máximo septentrional como estrella del crepúscu- 
lo (Declinación Máxima Norte Venus Vespertino: DMNVV) solo se alcanza antes del solsticio de verano. En cambio, como estrella matutina (DM$\left.\mathrm{NVM},+24^{\circ}, 6\right)$ se produce poco después. El otro extremo de la posición de Venus, la declinación máxima sur como estrella del amanecer (Declinación Máxima Sur Venus Matutino: DMSVM) se alcanza después del solsticio de invierno. Finalmente, su declinación máxima sur como estrella vespertina (DMSVV, declinación de $-26^{\circ}, 5$ ) se alcanza antes del solsticio de invierno, entre el 29 de octubre y el 6 noviembre en un $81 \%$ de las ocasiones en los últimos 4.000 años (Šprajc 2013). Aclarados, pues, estos extremos referentes a los movimientos, ciclos y posiciones de Venus, podemos penetrar mejor en la hipótesis que relaciona con este planeta algunas de las orientaciones registradas en los santuarios hispanos de tiempos protohistóricos. Conviene tener presente también que la relación estacional de las máximas declinaciones de Venus no ha cambiado en los últimos milenios, por lo que hoy estamos en la misma situación que en la época estudiada.

En el edificio de Caura, y si atendemos al horizonte oeste, el Santuario I y el altar del Santuario III parecen estar orientados hacia la puesta de Venus cuando alcanza su posición más meridional sobre la esfera celeste. Esto suele acontecer, como hemos comentado anteriormente, entre finales de octubre y principios de noviembre, cuando el planeta se sitúa apenas unos grados al sur de donde se pondrá el Sol en dicho solsticio. Por el contrario, la fase IV de este templo, y supuestamente también la III, apuntarían hacia el ocaso solar del solsticio de invierno. Pero, si nos fijamos ahora en el horizonte este, la orientación astronómica del altar y del primer edificio -recordemos que es la misma- está peor definida y solo resulta marginalmente consistente con el orto lunar en el lunasticio mayor norte (SLMN) y con el nacimiento de Venus en la declinación máxima norte como estrella matutina (DMNVM). Como hemos adelantado, esta última posición se produce poco después del solsticio de verano, entre el 30 de junio y el 19 de julio (Sprajc 2013). Hacia el este, la orientación del Santuario IV, e hipotéticamente la del III -también la misma en ambos casos-, enfocaría hacia la salida del Sol en el solsticio de verano. Considerando solo la dirección de los ocasos, los resultados de Caura podrían sugerir una posible evolución de la orientación astral (y quizás del ritual) en el santuario: centrado en Venus al inicio y en el Sol después. A día de hoy, esta hipótesis podría confirmarse si se obtuvieran resultados análogos en otros yacimientos similares o por argumentos obtenidos por vías independientes.

Para el Carambolo disponemos de las medidas de tres edificios orientados de forma similar pero con diferencias significativas (Tab. 1). Para analizar las orientaciones en este yacimiento hemos considerado, como en el resto de sitios, las dos direcciones posibles: este y oeste y además, en el caso del horizonte oriental, dos valores para la altura. Uno, $h=0^{\circ}$, se calculó suponiendo ortos sobre el horizonte lejano, que es básicamente plano. El segundo corresponde a la cumbre del cerro que, situada un poco al este de la puerta de este primer santuario, presentaría una altura del orden de $2^{\circ}$ para un observador colocado de pie sobre una superficie situada a la misma altura que la base del edificio y en su puerta. Estudiaremos los resultados considerando ambas posibilidades.

Con $h=0^{\circ}$, la estancia A-46, es decir, la capilla sur del Carambolo V, que indica la orientación del santuario original, apunta en dirección este hacia el orto del Sol en el solsticio de verano (SSV), además de a la DMNVM. De haberse perseguido este punto del horizonte, este dato resultaría de la mayor importancia, porque revelaría que los constructores del complejo sabían calcular la orientación con operaciones de cálculo que no requerían disponer de la observación del astro buscado. Ello si no se utilizó el punto opuesto del eje que, libre de obstáculos, era observable de forma directa. De hecho, en dirección oeste el edificio miraría en cambio hacia el PSI. En cambio la relación con la SSV deja de ser precisa, si se asume la presencia de la corona geológica del cerro original del Carambolo por delante de la puerta de este primer santuario, dando así pie a que $h=2^{\circ}$.

Hacia el este, la estancia A-40, que albergaba el altar taurodérmico y que funciona como capilla sur de las fases Carambolo IV y III, muestra una orientación consistente con la salida de la Luna en el lunasticio mayor norte (SLMN). Sin embargo, cuando giramos $180^{\circ}$ y enfilamos el horizonte occidental, la relación con el lunasticio (Puesta Lunasticio Mayor Sur: PLMS) pasa a ser marginal (ligeramente fuera de los errores), precisándose mucho más los vínculos con la puesta de Venus 
en su declinación máxima sur como estrella vespertina (DMSVV).

Finalmente, en el caso de la estancia A-1, aunque su orientación es cercana a la línea de los solsticios, solo encontramos una relación precisa hacia la SSV cuando consideramos $h=2^{\circ}$. Ello sugiere que, de contar con un encuadre astronómico intencionado, este podría haber sido meramente simbólico, pues la disposición del templo Carambolo V demuestra que la búsqueda efectiva y precisa del eje solsticial era posible y dominada por los constructores. De lo contrario, solo cabe pensar que esta sala A-1, atribuida al culto de Astarté y que se mantiene prácticamente invariable en las fases IV, III, II y I del santuario, se levantó en tal posición por dotar de simetría al edificio que sustituye al templo original (Escacena 2010: 110-111). De haberse perseguido cierta orientación simbólica, podría considerarse que los fenicios, autores de este recinto religioso, tuvieron tal vez en mente su creencia de que el Sol nacía en una montaña sagrada, Sapanu, de la que dejaron abundante iconografía.

La estructura carmonense de Saltillo, conocida como 'Ámbito 6', la que proporcionó los materiales arqueológicos más singulares y simbólicos (pithoi con decoración figurativa, cucharas de marfil, etc.), hacia el horizonte este, el eje mayor de la estancia se orienta en dirección a la salida lunar en el lunasticio mayor norte (SLMN), mientras que hacia el oeste apunta a la puesta en el lunasticio mayor sur (PLMS) y también, aunque ligeramente fuera de las incertidumbres de la medida, al ocaso de Venus en su máxima declinación sur (DMSVV). Como vemos, se producen aquí unas condiciones similares a las observadas en algunas fases de los santuarios de Caura y del Carambolo. Tales situaciones presentarían una relación lunar hacia el este y venusina hacia el oeste, aunque en Saltillo la precisión es menor y la relación hacia poniente podría ser también con la Luna.

Por último, la estancia IIIJ1 del poblado del Oral, relativamente alejado del área nuclear tartésica en sus aspectos geográficos y cronológicos, tiene la orientación característica de la 'familia de $55^{\circ}$. Hacia el este, sobre un horizonte muy cercano (unos centenares de metros) y que posiblemente haya variado algo con el tiempo, el edificio solo tiene una relación marginal con el orto lunar en el lunasticio mayor norte (SLMN).
Sin embargo, hacia el oeste la relación con la puesta de Venus en su momento de máxima declinación sur como estrella de la tarde (DMSVV) es bastante más precisa. En el apartado 4 recogimos la llamativa posición hacia el oeste, justo en la dirección a la que apunta el edificio, de una montaña lejana de perfil conspicuo sobre la que se produciría el ocaso de Venus en el DMSVV (Fig. 8). Estos dos hechos, la presencia de la montaña y la relación astronómica con el DMSVV, son importantes argumentos para establecer la dirección oeste como la relevante en la orientación del edificio. Consecuentemente, ello implica a Venus como astro de interés ritual.

\section{LA 'FAMILIA DE $55^{\circ}$ ' Y SUS IMPLICACIONES SOBRE EL CULTO}

Es posible que, en la precisión que estamos pidiendo a los edificios religiosos antiguos para establecer relaciones astronómicas claras, seamos más exigentes que en la requerida por sus propios constructores para los rituales de la época. En tal caso, las orientaciones celestes que los arquitectos y sacerdotes buscaron para sus templos y altares serían solo 'aproximadas', al primar lo simbólico sobre la exactitud matemática. Este extremo se ha planteado en relación al menos con el Carambolo (Escacena 2009: 99, 2011: 172). De ser así, los lazos helíacos podrían quedar reforzados sobre los demás, principalmente porque el Sol, sin duda el astro más importante de nuestro sistema planetario, ocupó la posición jerárquica superior en los panteones cósmicos del Mediterráneo antiguo. También hoy, cuando disponemos de sofisticados aparatos que pueden procurarnos una enorme precisión, los templos cristianos de cualquier ciudad se adaptan en ocasiones más al viario urbano preexistente que al dogma religioso estricto, aunque la primera condición impida respetar por completo la norma recogida en el segundo. La fuerza del símbolo se impone sobre la pulcritud de la medida. Aun así, la regularidad que muestra la 'familia de $55^{\circ}$ ' en los lugares protohistóricos aquí analizados podría entenderse como producto de una cierta preocupación por la precisión en la orientación de los edificios de culto.

Antes hemos señalado que las relaciones astronómicas de los santuarios de la 'familia de $55^{\circ}$, 
difieren si consideramos los ortos o los ocasos como fenómenos de referencia en el ritual. Cuando se presta atención a los ortos, los resultados destacan los aspectos lunares. Son coherentes con el punto de la salida lunar en el lunasticio mayor norte (SLMN), principalmente la estancia A-40 del Carambolo, el 'Ámbito 6' del edificio de Saltillo y, ligeramente fuera de la incertidumbre de las medidas, el altar de Caura y la estructura IIIJ1 del Oral. El lunasticio mayor norte se alcanza cada 18,6 años (el denominado periodo de regresión de los nodos) en la luna llena más cercana al solsticio de invierno. Abundando en este tema, no puede olvidarse que en el mundo cananeo existieron celebraciones religiosas vinculadas con la Luna (Olmo 1989), aunque los ciclos regulares de nuestro satélite se utilizaron más que nada para ordenar los meses y para inaugurar el año oficial (Olmo 1995a: 115, 1995b: 282), apertura que coincidía con la del año agrícola durante la luna de octubre (Stieglitz 2000: 695). La larga duración del periodo lunar de regresión de los nodos, tan amplio si se compara con los cursos solsticiales por ejemplo, impediría relacionar esta posición de la Luna con fiestas del mundo fenicio que sabemos de periodicidad más corta como el calendario religioso anual. Parece más prudente, entonces, buscar ataduras con astros que presenten periodos más breves, sobre todo si contamos con explicaciones distintas que entren en el margen de error aceptable de las medidas.

Primar por el contrario la orientación opuesta, la que involucra a los ocasos, convierte la declinación máxima sur de Venus como estrella vespertina (DMSVV) en el principal fenómeno astronómico de referencia para el altar de Coria del Río, para la capilla A-40 del Carambolo, para la construcción IIIJ1 del Oral y, ligeramente fuera de los márgenes de error extremos, también para Saltillo. En el apartado 5 señalamos que la DMSVV se produce cada 8 años, generalmente entre finales de octubre y principios de noviembre y apenas unos grados al sur de donde se produce el ocaso del Sol en el solsticio de invierno. Por lo tanto, ambos fenómenos son cercanos en el espacio y en el tiempo. La posición extrema de Venus, por así decir, precedería o 'anunciaría' la llegada del solsticio invernal. En tal caso contaríamos con ciclos festivos de más corta duración que los relacionables con la regresión de los nodos lunares, pero aún así difíciles de conectar con la liturgia fenicia conocida. De hecho, tal 'anunciación' del solsticio de diciembre solo acontecería cada 8 años, mientras que el ocaso solar por ese punto es un acontecimiento de periodicidad anual. Es más, si esa orientación supusiera la búsqueda de la DMSVV como 'buena nueva' reveladora del cercano nacimiento de un dios asimilable al Sol e hijo de la diosa, habría que concluir que esa divinidad masculina también nacía cada 8 años, algo no concordante con lo que sabemos de Baal como numen solar. Si su muerte y resurrección era una fiesta anual vinculada al solsticio de verano (Escacena 2007, 2009), es fácil asumir, a pesar de la carencia de datos, que su nacimiento se conmemorara en el momento en que la luz solar, epifanía de Baal en tanto que astro rey, empieza a crecer en su duración cotidiana a partir del solsticio de invierno.

El Sol saldría beneficiado si las orientaciones tuvieran intenciones simbólicas más que exactas, primando lo 'aproximado' sobre lo 'preciso'. A partir de ambas hipótesis se podrían buscar los argumentos adicionales para llegar a la mejor explicación.

Tres argumentos apoyarían la primera orientación, favorecedora del este y por tanto con vínculos lunares. El primero es la existencia en el lado menor, oriental, del altar de Caura de un cubículo donde se colocaba una muestra de sangre de los animales sacrificados. Los altares en forma de piel de toro hallados en Málaga muestran la misma preferencia por el flanco oriental donde también llevan el receptáculo para la sangre (Arancibia y Escalante 2006: 341). El segundo es la posición 'privilegiada' del costado oriental de los altares junto a la asherah. Esta posición del árbol sagrado respecto del altar, o de este en relación con aquel, está constatada en los santuarios españoles del Carambolo y de Caura (Escacena y Coto 2010: 171-172), así como en el templo portugués de Castro dos Ratinhos (Prados 2010: 268-269). El tercero es que en la fachada oriental se colocan casi todas las entradas a los recintos de culto de la época. En Coria del Río este rasgo no está confirmado, pero sí en santuarios excavados por completo como en el Carambolo (Fernández Flores y Rodríguez Azogue 2007: fig. 8 y 31) y en Cancho Roano (Celestino 2001: fig. 24).

En cambio, a favor de la hipótesis venusina, la que asumiría un papel preponderante de la orientación oeste, podrían argumentarse dos razones.

Trab. Prehist., 70, N. ${ }^{\circ}$ 1, enero-junio 2013, pp 114-139, ISSN: 0082-5638

doi: $10.3989 /$ tp.2013.12105 
La primera es que en Caura solo el horizonte occidental estaba lo suficientemente despejado de construcciones como para permitir una comprobación empírica de los correspondientes alineamientos celestes. La segunda es que el compartimento IIIJ1 del Oral muestra una orientación astronómica más precisa con Venus, cuyo ocaso se produce además sobre un elemento llamativo del horizonte lejano que podría haber sido utilizado como marcador astronómico.

Las hipótesis comentadas no deberían tomarse en principio como contradictorias y excluyentes. Es más, siempre puede sumarse a ambas el papel primordial del Sol como una tercera vía explicativa. Los edificios tienen las orientaciones adecuadas para sustentar todas estas posibilidades si las consideramos simplemente simbólicas y, por tanto, no muy precisas. Tampoco sería extraño que en cada caso hubiese que acudir a una explicación particular de las tres barajadas: orientaciones solares, lunares o venusinas. Aquí queda aún mucha tarea por realizar. De hecho, aunque los citados altares taurodérmicos de Málaga parecen privilegiar también el este, sus ejes longitudinales buscan más bien una orientación sureste-noroeste, diferente por tanto de la disposición noreste-suroeste del ara de Coria del Río (7).

Volviendo a la hipótesis que, sin excluir el este, tiene en cuenta la orientación hacia el occidente, habría que señalar que a esta última dirección enfoca el extremo oeste del eje longitudinal del Carambolo V, el templo más antiguo de este complejo ceremonial. Otro tanto sucede con la pared perimetral septentrional del Santuario IV de Coria del Río y el templo de su fase III, no excavado, si estuviese en la misma vertical que el muro del recinto IV inmediatamente posterior. Se trataría ahora de una orientación consistente con el ocaso solar durante el solsticio de invierno. Por ello, tal vez la conclusión más correcta sea proponer que el análisis arqueoastronómico apoya la tesis, ya asumida con anterioridad para estos templos (Escacena 2010: 111), de que rendían culto al dios Baal/Melqart y a la divinidad femenina Astarté,

(7) Los altares de Málaga, localizados en un santuario fenicio de la $\mathrm{C} /$ Císter y construidos con las mismas características cromáticas que el de Caura, no han sido medidos astronómicamente. Las referencias de orientación que comentamos proceden solo de los planos publicados por sus excavadores. Entre nuestras prioridades está el estudio arqueoastronómico de este yacimiento en el futuro. personificaciones fenicias de la pareja de astros formada por Sol y por Venus. En la arquitectura del Carambolo, este culto dual habría dejado su más directo reflejo en las dos capillas que funcionaron a la vez en los distintos momentos de vida del santuario (Fernández Flores y Rodríguez Azogue 2007: 228-229). Según dichos autores, el altar con forma de piel de toro sugiere la dedicación de la estancia A-40 a Baal (Fernández Flores y Rodríguez Azogue 2005a: 136-137). En este contexto litúrgico, habrían sido especialmente importantes las fiestas relacionadas con el ciclo solar. Este habría supuesto, de hecho, la base de la creencia en un dios que muere y que resucita al tercer día, cuyo trasfondo astronómico estaba inspirado en la parada solsticial y en la inmediata recuperación del desplazamiento solar sobre el horizonte en la tercera jornada de este evento helíaco (Escacena 2007, 2009). Como hemos visto en el presente trabajo, la evidencia astronómica manifiesta una clara relación solsticial (solar) para el santuario inicial del Carambolo. En cambio, las orientaciones de las fases posteriores del edificio (Carambolo IV y III) ofrecen más problemas para establecer una clara relación con un hecho astronómico concreto.

De certificarse que en otros lugares hay más edificios religiosos de la 'familia de 55', grupo relacionable posiblemente más con Venus que con el Sol, tendríamos el indicador de su dedicación a Astarté. Como es bien sabido, las fuentes clásicas aluden a enclaves y templos costeros consagrados a Venus/Afrodita en la costa de la Península Ibérica, sitios que tradicionalmente se han relacionado con Astarté (Pérez López 1998; Marín 2010: 492). Un lugar especialmente interesante en este sentido, con información publicada pero insuficiente para precisar su orientación astronómica, es el de la Algaida, en Sanlúcar de Barrameda (Cádiz). Este sitio, posterior a la mayor parte de los aquí estudiados, se inserta en realidad en la misma tradición religiosa. Blanco y Corzo (1983: 123) lo han identificado con las referencias de Estrabón (III 1, 9) al templo de Phosphóros, que porta luz, y con el epíteto latino lux dubia, luz dudosa o incierta, crepúsculo. Para Corzo (2000: 147-150), el culto que se llevaba a cabo en el santuario de la Algaida sería de tipo auroral, relacionado con una deidad, protectora de la navegación y de la fecundidad femenina, e identificada con Astarté como lucero de la mañana. De hecho, son mu- 
chos los autores que han relacionado Phosphóros con Astarté, luego asimilada a la Venus romana y personificada en el planeta homónimo (Tovar 1962: 814; Salinas 1988).

\section{AGRADECIMIENTOS}

Antes o durante el trabajo de campo contamos con la colaboración de los arqueólogos involucrados en la excavación de algunos de los santuarios estudiados, como Araceli Rodríguez Azogue, Álvaro Fernández Flores, Rocío Izquierdo de Montes, María Belén Deamos, Jesús Fernández Jurado y Feliciana Sala Sellés. A todos ellos agradecemos sinceramente su ayuda. Parte de este trabajo contó con la financiación del proyecto "Arqueoastronomía" (código 7/93) del Instituto de Astrofísica de Canarias. Otros gastos corrieron a cargo de los proyectos HUM2007-63419/HIST y HAR200801119 (Ministerio Español de Ciencia y Tecnología) y del Grupo HUM-402 del III Plan Andaluz de Investigación (Junta de Andalucía).

\section{BIBLIOGRAFÍA}

Abad, L. y Sala, F. 1993: El poblado ibérico de El Oral (San Fulgencio, Alicante). Trabajos Varios del Servicio de Investigaciones Prehistóricas 90, Diputación de Valencia. Valencia.

Almagro-Gorbea, M. y Gran-Aymerich, J. M. J. 1991: "El estanque monumental de Bibracte (Mont Beuvray-Borgoña)". Complutum Extra 1: 1-248.

Amores, F. y Escacena, J. L. 2003: "De toros y de tesoros: simbología y función de las joyas de El Carambolo". En A. García-Baquero y P. Romero (eds.): Fiestas de toros y sociedad. Universidad de Sevilla. Sevilla: 41-68.

Aparicio, A.; Esteban, C. y Belmonte, J. A. 2000: "Las bases astronómicas". En J. A. Belmonte (ed.): $A r$ queoastronomía Hispana. Equipo Sirius. Madrid: 19-65.

Arancibia, A. y Escalante, M. M. 2006: "La Málaga fenicio-púnica a la luz de los últimos hallazgos". Mainake XXVIII: 333-360.

Armentia, J. E.; Almagro-Gorbea, M.; Gran-Aymerich, J. M. J. y Rodríguez, G. 1993: "Trazado y orientación topo-astronómica del Estanque Monumental de Bibracte". En J. Mangas y J. Alvar (eds.): Homenaje a José María Blázquez I. Ediciones Clásicas, Madrid: 267-284.
Arteaga, O.; Schulz, H. D. y Roos, A. M. 1995: "El problema del 'Lacus Ligustinus'. Investigaciones geoarqueológicas en torno a las Marismas del Bajo Guadalquivir". Tartessos 25 años después, 1968-1993, Jerez de la Frontera. Congreso Conmemorativo del $V$ Symposium Internacional de Prehistoria Peninsular (1993 Cádiz): 99-135. Jerez de la Frontera.

Aubet, M. E. 1975: La necrópolis de Setefilla en Lora del Río, Sevilla. Programa de Investigaciones Protohistóricas 2, CSIC, Universidad de Barcelona. Barcelona.

Aubet, M. E. 1978: La necrópolis de Setefilla en Lora del Río, Sevilla (túmulo B). CSIC, Universidad de Barcelona. Barcelona.

Aubet, M. E.; Serna, M. R.; Escacena, J. L. y Ruiz Delgado, M. M. 1983: La Mesa de Setefilla. Lora del Río (Sevilla). Campaña de 1979. Excavaciones Arqueológicas en España 122, Ministerio de Cultura. Madrid.

Aveni, A. F. 1991: Observadores del cielo en el México antiguo. Fondo de Cultura Económica. México D.F.

Baquedano, M. I. y Escoraza, C. M. 1998: "Alineaciones astronómicas en la necrópolis de la Edad del Hierro de la Osera (Chamartín de la Sierra, Ávila)". Complutum 9: 85-100.

Belén, M. 1993: "Mil años de historia de Coria: la ciudad prerromana". En J. L. Escacena (ed.): $A r-$ queología de Coria del Río y su entorno. Azotea. Revista de Cultura del Ayuntamiento de Coria del Río 11-12: 35-60.

Belén, M. 2000: "Itinerarios arqueológicos por la geografía sagrada del Extremo Occidente". En J. H. Fernández Gómez y B. Costa Ribas (eds.): Santuarios fenicio-púnicos en Iberia y su influencia en los cultos indígenas. XIV Jornadas de Arqueología Fenicio-Púnica, Ibiza 1999. Trabajos del Museo Arqueológico de Ibiza y Formentera 46: 57-102.

Belén, M. 2001: "Arquitectura religiosa orientalizante en el Bajo Guadalquivir". En D. Ruiz Mata y S. Celestino (eds.): Arquitectura oriental y orientalizante en la Península Ibérica. Centro de Estudios de Próximo Oriente, CSIC. Madrid: 1-16.

Belén, M.; Anglada, R.; Escacena, J. L.; Jiménez, A.; Lineros, R y Rodríguez, I. 1997: Arqueología en Carmona (Sevilla). Excavaciones en la Casa-Palacio del Marqués de Saltillo. Junta de Andalucía. Sevilla.

Belén, M y Escacena, J. L. 2002: "La imagen de la divinidad en el mundo tartésico". En E. Ferrer (ed.): Ex Oriente Lux: las religiones orientales antiguas en la Península Ibérica. Universidad de Sevilla. Sevilla: $159-184$.

Belmonte, J. A. 1999: Las leyes del cielo. Astronomía y civilizaciones antiguas. Temas de Hoy. Madrid.

Belmonte, J. A. 2009: "La arqueoastronomía en Europa: la singularidad del caso español". Complutum 20 (2): 55-67.

Blanco, A. 1968: "Los primeros ensayos de representación plástica de la figura humana en el arte español". España en las crisis del arte europeo. Coloquios ce-

Trab. Prehist., 70, N. ${ }^{\circ}$ 1, enero-junio 2013, pp 114-139, ISSN: 0082-5638

doi: 10.3989/tp.2013.12105 
lebrados en conmemoración de los XXV años de la Fundación del Consejo Superior de Investigaciones Cientificas. Casa de Velázquez. Madrid: 21-28.

Blanco, A. 1979: Historia de Sevilla. I. La ciudad antigua (desde la Prehistoria a los Visigodos). Universidad de Sevilla. Sevilla.

Blanco, A. y Corzo, R. 1983: "Monte Algaida. Un santuario en la desembocadura del Guadalquivir". Historia 16 (87): 123-128.

Blanco, A. y Rothenberg, B. 1981: Exploración arqueometalúrgica de Huelva (EAH). Riotinto Minera-Labor. Barcelona.

Bonsor, G. 1899: "Les colonies agricoles pré-romaines de la vallée du Bétis". Revue Archéologique XXXV: 285-300.

Bonsor, G. y Thouvenot, R. 1928: "Nécropole ibérique de Setefilla, Lora del Río (Sevilla). Fouilles de 19261927". Bibliothèque de l'École des Hautes Études Hispaniques XIV.

Borja, F. 2010: "El Carambolo. Aproximación geoarqueológica". En M. L. de la Bandera y E. Ferrer (ed.): El Carambolo. 50 años de un tesoro. Universidad de Sevilla. Sevilla: 177-201.

Carriazo, J. de M. 1973: Tartesos y el Carambolo. Investigaciones arqueológicas sobre la Protohistoria de la Baja Andalucía. Ministerio de Educación y Ciencia. Madrid.

Castro, P. V.; Lull, V. y Micó, R. 1996: Cronología de la prehistoria reciente de la Península Ibérica y Baleares (c. 2800-900 cal ANE). British Archaeological Reports, International Series 652, Archaeopress. Oxford.

Celestino, S. 1994: "Los altares en forma de "lingote chipriota' de los santuarios de Cancho Roano". Revista de Estudios Ibéricos 1. La escultura ibérica: 291-309.

Celestino, S. 1997: "Santuarios, centros comerciales y paisajes sacros". Quaderns de Prehistòria i Arqueologia de Castelló 18: 359-389.

Celestino, S. 2001: "Los santuarios de Cancho Roano. Del indigenismo al orientalismo arquitectónico". En D. Ruiz Mata y S. Celestino (eds.): Arquitectura oriental y orientalizante en la Península Ibérica. Centro de Estudios de Próximo Oriente, CSIC. Madrid: $17-56$.

Celestino, S. 2008: "Los altares en forma de piel de toro de la Península Ibérica". En J. Justel, J. P. Vita y J. A. Zamora (eds.): Las culturas del Próximo Oriente antiguo y su expansión mediterránea. Instituto de Estudios Islámicos y del Próximo Oriente. Zaragoza: 321-348.

Cerdeño, M. y Rodríguez, G. 2009: “Arqueoastronomía: una nueva perspectiva en la investigación arqueológica”. Complutum 20 (2): 11-21.

Cerdeño, M. L.; Rodríguez, G. y Folgueira, M. 20012002: "El paisaje funerario de la cultura celtibérica". Anales de Prehistoria y Arqueología de la Universidad de Murcia 16-17: 253-261.
Cerdeño, M. L.; Rodríguez, G.; Folgueira, M. y Hernández, M. C. 2004: "Novedades culturales y metodológicas en la necrópolis de Herrería (Guadalajara)". En M. Barril y A. Rodero (eds.): Novedades Arqueológicas Celtibéricas. Museo Arqueológico Nacional. Madrid: 43-62.

Cerdeño, M. L.; Rodríguez, G.; Moya, P.; Ibarra, A. y Herrero, S. 2006: "Los estudios de arqueoastronomía en España: estado de la cuestión". Trabajos de Prehistoria 63 (2): 13-34.

Conde, M.; Izquierdo, R. y Escacena, J. L. 2005: “Dos escarabeos del santuario fenicio de Caura en su contexto histórico y arqueológico". Spal 14: 75-89.

Corzo, R. 2000: "El santuario de La Algaida (Sanlúcar de Barrameda, Cádiz) y la formación de los talleres artesanales". En J. H. Fernández Gómez y B. Costa Ribas (eds.): Santuarios fenicio-púnicos en Iberia y su influencia en los cultos indígenas. XIV Jornadas de Arqueología Fenicio-Púnica (Ibiza 1999). Trabajos del Museo Arqueológico de Ibiza y Formentera 46: 147-183.

Escacena, J. L. 2001: "Fenicios a las puertas de Tartessos". Complutum 12: 73-96.

Escacena, J. L. 2005: "Darwin y Tartessos". En S. Celestino y J. Jiménez (eds.): El Periodo Orientalizante. Anejos de Archivo Español de Arqueología XXXV, CSIC, Mérida: 189-219.

Escacena, J. L. 2007: "El dios que resucita: claves de un mito en su primer viaje a Occidente". En J. J. Justel, B. E. Solans, J. P. Vita y J. Á. Zamora (eds.): Las aguas primigenias. El Próximo Oriente Antiguo como fuente de civilización. Actas del IV Congreso Español de Antiguo Oriente Próximo (Zaragoza 2006): 615-651. Zaragoza.

Escacena, J. L. 2009: "La Égersis de Melqart. Hipótesis sobre una teología solar cananea". Complutum 20 (2): 95-120.

Escacena, J. L. 2010: "El Carambolo y la construcción de la arqueología tartésica". En M. L. de la Bandera y E. Ferrer (ed.): El Carambolo. 50 años de un tesoro. Universidad de Sevilla. Sevilla: 99-148.

Escacena, J. L. 2011: "Variación identitaria entre los orientales de Tartessos. Reflexiones desde el antiesencialismo darwinista". En M. Álvarez (ed.): Fenicios en Tartessos: nuevas perspectivas. British Archaeological Reports, International Series 2245, Archaeopress. Oxford: 161-192.

Escacena, J. L. y Coto, M. 2010: "Altares para la eternidad". Spal 19: 149-185.

Escacena, J. L.; Fernández Flores, A. y Rodríguez Azogue, A. 2007: "Sobre el Carambolo: un híppos sagrado del santuario IV y su contexto arqueológico". Archivo Español de Arqueología 80: 5-27.

Escacena, J. L. e Izquierdo, R. 2000: "Altares para Baal". Arys 3: 11-40.

Escacena, J. L. e Izquierdo, R. 2001: “Oriente en Occidente. Arquitectura civil y religiosa en un barrio fenicio de la Caura tartésica". En D. Ruiz Mata y 
S. Celestino (eds.): Arquitectura oriental y orientalizante en la Península Ibérica Centro de Estudios del Próximo Oriente, CSIC. Madrid: 123-157.

Escacena, J. L. e Izquierdo, R. 2008: “A propósito del paisaje sagrado fenicio de la paleodesembocadura del Guadalquivir". En X. Dupré, S. Ribichini y S. Verger (eds.): Saturnia Tellus. Definizioni dello spazio consacrato in ambiente etrusco, italico, feniciopunico, iberico e celtico. Convegno internazionale di studi (Roma 2004): 431-455. Roma.

Escacena, J. L. y Vázquez, M. I. 2009: "Conchas de salvación". Spal 18: 51-82.

Esteban, C. 2002: "Elementos astronómicos en el mundo religioso y funerario ibérico". Trabajos de Prehistoria 59 (2): 81-100.

Esteban, C. 2003: "Temples and astronomy in Carthage". En M. Blomberg, P. E. Blomberg y G. Henriksson (eds.): Calendars, symbols, and orientations: legacies of astronomy in culture proceedings of the 9th annual meeting of the European Society for Astronomy in Culture (SEAC) (Estocolmo 2001). Uppsala Astronomical Observatory Report 59: 135-142.

Esteban, C. 2009: "La astronomía cultural ¿es interdisciplinar? Reflexiones de un astrofísico". Complutum 20 (2): 69-77.

Esteban, C. 2012: "Orientación de las tumbas y astronomía en la necrópolis de la Angorrilla". En A. Fernández Flores, A. Rodríguez Azogue y M. Casado Ariza (eds.): La necrópolis de época tartésica de la Angorrilla (Alcalá del Río, Sevilla). Publicaciones de la Universidad de Sevilla. En prensa.

Esteban, C. y Cortell, E. 1997: "Consideraciones arqueoastronómicas sobre el santuario ibérico de La Serreta". Recerques del Museu d'Alcoi 6: 131-140.

Esteban, C. y Delgado, M. 2005: "Sobre el análisis arqueoastronómico de dos yacimientos tinerfeños y la importancia de los equinoccios en el ritual aborigen". Tabona 13: 187-214.

Esteban, C. y Moret, S. 2006: "Ciclos de tiempo en la cultura ibérica: la orientación astronómica del Templo del Tossal de Sant Miquel de Llíria". Trabajos de Prehistoria 63 (1): 167-178.

Fernández Flores, A. y Rodríguez Azogue, A. 2005a: "El complejo monumental del Carambolo Alto, Camas (Sevilla). Un santuario orientalizante en la paleodesembocadura del Guadalquivir". Trabajos de Prehistoria 62 (1): 111-138.

Fernández Flores, A. y Rodríguez Azogue, A. 2005b: "Nuevas excavaciones en el Carambolo Alto, Camas (Sevilla). Resultados preliminares". En S. Celestino y J. Jiménez (eds.): El Periodo Orientalizante. Anejos de Archivo Español de Arqueología XXXV, CSIC. Mérida: 843-862.

Fernández Flores, A. y Rodríguez Azogue, A. 2007: Tartessos desvelado. La colonización fenicia del suroeste peninsular y el origen y ocaso de Tartessos. Almuzara. Córdoba.
Fernández Jurado, J. 1987: “Tejada la Vieja: una ciudad protohistórica”. Huelva Arqueológica 9: 9-170.

Fernández Jurado, J. y García Sanz, C. 2001: “Arquitectura orientalizante en Huelva". En D. Ruiz Mata y S. Celestino (eds.): Arquitectura oriental y orientalizante en la Península Ibérica. Centro de Estudios de Próximo Oriente, CSIC. Madrid: 159-171.

González García, A. C. 2009: "Análisis estadístico de las orientaciones de los megalitos de la Península Ibérica”. Complutum 20 (2): 177-186.

González García, A. C.; Costa, L.; Zedda, M. P. y Belmonte, J. A. 2007: "The orientation of the Punic tombs of Ibiza and Sardinia”. En M. P. Zedda y J. A. Belmonte (eds.): Light and shadows in Cultural Astronomy. Proceedings of the European Society for Astronomy in Culture, SEAC (2005 Isili, Sardinia): 47-57. Isili.

Hoskin, M. A. 2001: Tombs, temples and their orientations. A new perspective on mediterranean Prehistory. Ocarina Books, Bognor Regis.

Hoskin, M. A. 2006: “¿Por qué algunas tumbas megalíticas europeas miran hacia el este y otras hacia el oeste?". En J. Lull (ed.): Trabajos de arqueoastronomía: ejemplos de Africa, América, Europa y Oceanía. Agrupación Astronómica de La Safor. Valencia: 131-142.

Iwaniszewski, S. 1994: "De la astroarqueología a la astronomía cultural". Trabajos de Prehistoria 51 (2): 5-20.

Izquierdo, R. 1998: "La cabaña circular en el mundo tartésico. Consideraciones sobre su uso como indicador étnico". Zephyrvs 51: 277-288.

Izquierdo, R. y Escacena, J. L. 1998: "Sobre El Carambolo: 'La trompeta de Argantonio'". Archivo Español de Arqueología 71: 27-36.

Ladrón de Guevara, I.; Sánchez, M.; Rodríguez de Zuloaga, M. y Lazarich, M. 1992: "Materiales inéditos de Setefilla (Lora del Río, Sevilla)”. Spal 1: 293-312.

López Plaza, M. S.; Alonso, F.; Cornide, M. y Álvarez, A. 1991-92: “Aplicación de la astronomía al estudio de la orientación de los sepulcros megalíticos de corredor en la zona noroccidental de la Península Ibérica". Zephyrvs XLIV-XLV: 183-192.

Marín, M. C. 2010: "Imagen y culto de Astarté en la Península Ibérica I: Las fuentes griegas y latinas". En M. L. de la Bandera y E. Ferrer (ed.): El Carambolo. 50 años de un tesoro. Universidad de Sevilla. Sevilla: 491-512.

Olmo, G. del 1989: "Rituales sacrificiales de plenilunio y novilunio (KTU 1.109/1.46)". Aula Orientalis VII (2): 181-188.

Olmo, G. del 1995a: "Mitología y religión de Siria en el II milenio a.C. (1500-1200)". En G. del Olmo (ed.): Mitología y religión del Oriente Antiguo. II/2, Semitas occidentales (Emar, Ugarit, Hebreos, Fenicios, Arameos, Árabes). Ausa. Sabadell: 45-222.

Olmo, G. del 1995b: "La religión cananea de los antiguos hebreos". En G. del Olmo (ed.): Mitología y religión del Oriente Antiguo. II/2, Semitas occiden-

Trab. Prehist., 70, N. ${ }^{\circ}$ 1, enero-junio 2013, pp 114-139, ISSN: 0082-5638

doi: $10.3989 /$ tp.2013.12105 
tales (Emar, Ugarit, Hebreos, Fenicios, Arameos, Árabes). Ausa. Sabadell: 223-350.

Pérez López, I. 1998: Los santuarios de la Baetica en la Antigüedad: los santuarios de la costa. Universidad de Cádiz. Cádiz.

Prados, F. 2010: "La arquitectura sagrada: un santuario del siglo IX a.C.”. En L. Berrocal-Rangel y A. C. Silva (eds.): $O$ Castro dos Ratinhos (Barragem do Algarve, Moura). Escavaçôes num povoado protohistórico do Guadiana, 2004-2007. O Arqueólogo Portugués, Suplemento 6, Museo Nacional de Arqueología. Lisboa: 259-276.

Ramos Sainz M. L. 1986: Estudio sobre el ritual funerario en las necrópolis fenicias y púnicas de la Península Ibérica. Universidad Autónoma de Madrid. Madrid.

Rodríguez Azogue, A. y Fernández Flores, A. 2005: "El santuario orientalizante del cerro del Carambolo, Camas (Sevilla). Avance de los resultados de la segunda fase de la intervención”. En S. Celestino y J. Jiménez (eds.): El Periodo Orientalizante. Anejos de Archivo Español de Arqueología XXXV, CSIC. Mérida: 863-871.

Rodríguez, G.; Cerdeño, M. L.; Folgueira, M. y Sagardoy, T. 2006: “Observaciones topoastronómicas en la zona arqueológica de El Ceremeño (Herrería, Guadalajara)". Complutum 17: 133-143.

Sala, F. y Abad, L. 2006: "Arquitectura monumental y arquitectura doméstica en la Contestania". Lvcentvm XXV: 23-46.

Salinas, M. 1988: “El 'Hieron Akroteríon' y la geografía religiosa del Extremo Occidente según Estrabón". En G. Pereira (ed.): Actas del $1^{\text {er }}$ Congreso Peninsular de Historia Antigua (Santiago de Compostela 1986) II: 135-147. Santiago de Compostela.

Sprajc, I. 1996: Venus, lluvia y maiz: simbolismo y astronomía en la cosmovisión mesoamericana. Instituto Nacional de Antropología e Historia. México D.F.

Sprajc, I. 2013: "Alignments upon Venus (and other planets): identification and analysis". En C.L.N. Ruggles (ed.): Handbook of Archaeoastronomy and Ethnoastronomy. Springer, Berlín: en prensa.

Stieglitz, R. R. 2000: "The phoenician-punic calendar". En M. Barthelemy y M. E. Aubet (eds.): IV Congreso Internacional de Estudios Fenicios y Púnicos (Cádiz 1995) II: 691-695. Cádiz.

Tovar, A. 1962: "Papeletas de Geografía Turdetana". Homenaje al profesor Cayetano de Mergelina. Universidad de Murcia. Murcia: 813-819. 